\title{
THE HODGE STRUCTURE OF SEMIAMPLE HYPERSURFACES AND A GENERALIZATION OF THE MONOMIAL-DIVISOR MIRROR MAP
}

\author{
ANVAR R. MAVLYUTOV
}

\begin{abstract}
We explicitly compute the cohomology ring of semiample nondegenerate hypersurfaces in complete simplicial toric varieties. The monomialdivisor mirror map is generalized to a map between the whole Picard group and the space of infinitesimal deformations for a mirror pair of Calabi-Yau hypersurfaces. This map is compatible with certain vanishing limiting products of the subrings of the chiral rings, on which the ring structure is related to a product of the roots of $A$-type Lie algebra.
\end{abstract}

\section{CONTEnTS}

1. A brief review of semiample divisors in toric varieties. 3

$\begin{array}{lll}2 . & \text { The Hodge structure of semiample nondegenerate hypersurfaces. } & 6\end{array}$

3. The Picard group of semiample hypersurfaces. 21

4. The B-model correlation functions. 23

5. A generalization of the monomial-divisor mirror map. 25

References

In this paper we solve the long-standing problem of describing the cohomology of semiample hypersurfaces in complete simplicial toric varieties. The problem became important when physicists found a relation between quantum field theory and the geometry of Calabi-Yau manifolds. Toric varieties used as an ambient space provide a large number of examples of Calabi-Yau manifolds realized as complete intersections. Cohomology of Calabi-Yau manifolds is interpreted as a finite dimensional subspace of the Hilbert space of states in physics. Physicists used the (mathematically non-rigorous) orbifold Landau-Ginzburg theory to compute the cohomology of Calabi-Yau manifolds in some examples coming from toric varieties (e.g., [V]). However, no general solution in physics was found. About ten years ago physicists discovered Mirror Symmetry, which started with a certain symmetry of the Hodge numbers of two topologically distinct Calabi-Yau manifolds. Soon after that, V. Batyrev in [B] generalized the construction of physicists using a duality of reflexive polytopes and Calabi-Yau hypersurfaces in toric varieties. Being the

1991 Mathematics Subject Classification. Primary: 14M25.

Key words and phrases. Toric geometry, mirror symmetry.

This research was conducted by the author for the Clay Mathematics Institute. 
resolutions of ample hypersurfaces, these mirror symmetric hypersurfaces are semiample. The problem to compute the cohomology is also relevant to the calculation of the B-model chiral ring since the cohomology is isomorphic to this ring.

In most discussions of the Mirror Theorems (see [CKa]), it is not mentioned that the results proved by Givental and Lian-Liu-Yau apply only to some (called toric and polynomial) parts of the A-model and B-model chiral rings of a mirror pair of Calabi-Yau manifolds. An explicit description of the full cohomology of the manifolds is a necessary part to extend their techniques to a complete solution.

The history of calculating the cohomology of hypersurfaces starts with a paper of P. Griffiths (see [G]). There he found the residue (primitive) part of the middle cohomology of smooth hypersurfaces in a projective space. Such hypersurfaces are automatically ample, implying certain vanishing theorems which allow to do the calculations. In a later paper $\mathrm{CaG}$, the product structure was computed on the residue part. Using essentially the same method, V. Batyrev and D. Cox in BC described the residue part of the middle cohomology of ample quasismooth hypersurfaces in complete simplicial toric varieties, for which the analogous vanishing theorems hold as well. For semiample hypersurfaces the vanishing theorems do not hold - that is where the complexity of the problem arises. However, the paper $B$ also contained an alternative method of finding the description for an open subset of ample quasismooth hypersurfaces which consists of nondegenerate (transversal to the torus orbits) hypersurfaces. In this case, the Gysin spectral sequence together with the ampleness have been used. We noticed that this spectral sequence can be applied to the semiample nondegenerate hypersurfaces as well, but because of the missing ampleness property the complexity still appears. While this paper provides a solution only for the semiample nondegenerate hypersurfaces, we said in [M3 that our strategy is to use this solution in order to find an answer to the question for all semiample quasismooth hypersurfaces. The answer should be very similar since the semiample quasismooth hypersurfaces are flat deformations of the nondegenerate ones.

Here is the plan of our paper. In Section f w we start with introducing the notation, definitions, and then review some basic results from [M2].

Section 2 is devoted to deriving our main result: an explicit description of the cohomology ring of semiample nondegenerate hypersurfaces in complete simplicial toric varieties. In M2 we showed that this cohomology splits into the toric and residue parts. The toric part is the image of cohomology of the ambient space, while its complement comes from the residues of rational differential forms with poles along the hypersurface. The toric part of the cohomology has already been calculated, and we follow the algorithmic approach suggested in [M2] to derive the residue part. In parallel, we also compute the product structure on the cohomology.

In Section 3, we apply the results from Section 2 to find the Picard group of semiample nondegenerate hypersurfaces. We give two alternative descriptions of the Picard group, one of which is algebraic and the other is geometric. A previous approach of [B] to compute the Picard group has gaps in the proof, complicated restrictions and not quite explicit (the toric variety $\mathbb{P}_{\left(\Sigma_{0}, L\right)}$ in the proof of [ $\mathbb{B}$, Theorem 2] is not necessarily simplicial, hence [B, Proposition 4] could not be used in this case).

In Section 0 , we use one of the main results from [M2] to describe a subring of $H^{*}\left(X, \wedge^{*} \mathcal{T}_{X}\right)$ of semiample minimal Calabi-Yau hypersurfaces in toric varieties, 
which contains $H^{1}\left(X, \mathcal{T}_{X}\right)$. Here, $\wedge^{*} \mathcal{T}_{X}$ is the Zariski $p$-th exterior power of the tangent sheaf, introduced in [M2]. We discover that the remarkable product structure on the subring is closely related to a product of the roots of an $A$-type Lie algebra. Looking at the subring of the cohomology of semiample minimal Calabi-Yau hypersurfaces, generated by the Picard group, we find that a very similar product structure takes place.

Using the above, in Section 5 , we propose a generalization of the monomialdivisor mirror map of Aspinwall, Greene and Morrison, which is supposed to be the derivative of the mirror map at the large radius limit points. Our map is supported by a compatibility of certain vanishing limiting products of the chiral rings of Calabi-Yau hypersurfaces in the Batyrev mirror construction. We also predict that the same vanishing occurs in the quantum cohomology of the CalabiYau hypersurfaces.

Acknowledgments. While all the essential techniques for solving this problem were developed in my Ph.D. dissertation (see [M3]), I found the last clue this summer being a Liftoff researcher of the Clay Mathematics Institute. I would like to thank David Cox for suggesting me this complicated and interesting problem for my dissertation research, and, also, for many helpful discussions and useful comments.

\section{A BRIEF REVIEW OF SEMIAMPLE DIVISORS IN TORIC VARIETIES.}

In this section we review some basic notation and facts from [M2] about semiample divisors in toric varieties.

Throughout this paper we will use the following dictionary:

$M$ is a lattice of rank $d$.

$N=\operatorname{Hom}(M, \mathbb{Z})$ is the dual lattice.

$M_{\mathbb{R}}$ and $N_{\mathbb{R}}$ are the $\mathbb{R}$-scalar extensions of $M$ and $N$, respectively.

$\Sigma$ is a finite rational (usually simplicial) fan in $N_{\mathbb{R}}$.

$\mathbf{P}_{\Sigma}$ is a $d$-dimensional toric variety associated with $\Sigma$.

$\mathbf{T}_{\sigma}$ is a torus corresponding to the cone $\sigma \in \Sigma$.

$V(\sigma)$ is a toric variety equal to the closure of $\mathbf{T}_{\sigma}$ in $\mathbf{P}_{\Sigma}$.

$\Sigma(k)$ is the set of all $k$-dimensional cones in $\Sigma$.

$\Sigma(1)=\left\{\rho_{1}, \ldots, \rho_{n}\right\}$ is the set of 1-dimensional cones in $\Sigma$ with the minimal integral generators $e_{1}, \ldots, e_{n}$, respectively.

$D_{i}$ is a torus invariant divisor in $\mathbf{P}_{\Sigma}$, corresponding to $\rho_{i}$.

$S=S\left(\mathbf{P}_{\Sigma}\right)=\mathbb{C}\left[x_{1}, \ldots, x_{n}\right]$ is the homogeneous coordinate ring of $\mathbf{P}_{\Sigma}$.

$\Delta_{D}=\left\{m \in M_{\mathbb{R}}:\left\langle m, e_{i}\right\rangle \geq-a_{i}\right.$ for all $\left.i\right\} \subset M_{\mathbb{R}}$ is a convex polytope of a Weil divisor $D=\sum_{i=1}^{n} a_{i} D_{i}$.

A Cartier divisor $D$ on $\mathbf{P}_{\Sigma}$ is called semiample if $\mathcal{O}_{\mathbf{P}_{\Sigma}}(D)$ is generated by global sections. The Iitaka dimension of a Cartier divisor $D$ on $\mathbf{P}_{\Sigma}$ is defined by

$$
\kappa(D):=\kappa\left(\mathcal{O}_{\mathbf{P}_{\Sigma}}(D)\right)=\operatorname{dim} \phi_{D}\left(\mathbf{P}_{\Sigma}\right)
$$

where $\phi_{D}: \mathbf{P}_{\Sigma} \rightarrow \mathbb{P}\left(H^{0}\left(\mathbf{P}_{\Sigma}, \mathcal{O}_{\mathbf{P}_{\Sigma}}(D)\right)\right)$ is the rational map defined by the sections of the line bundle $\mathcal{O}_{\mathbf{P}_{\Sigma}}(D)$. For a torus invariant $D, \kappa(D)$ coincides with the dimension of the associated polytope $\Delta_{D}$.

Definition 1.1. [M2] A semiample divisor $D$ on a complete toric variety $\mathbf{P}_{\Sigma}$ is called $i$-semiample if the Iitaka dimension $\kappa(D)=i$. 
The following result which describes the unique properties of the map associated with a semiample divisor is a simplification of Theorem 1.4 in [M2].

Theorem 1.2. Let $[D] \in A_{d-1}\left(\mathbf{P}_{\Sigma}\right)$ be an $i$-semiample divisor class on a complete toric variety $\mathbf{P}_{\Sigma}$ of dimension $d$. Then, there exists a unique complete toric variety $\mathbf{P}_{\Sigma_{D}}$ with a surjective morphism $\pi: \mathbf{P}_{\Sigma} \rightarrow \mathbf{P}_{\Sigma_{D}}$, arising from a surjective homomorphism of lattices $\tilde{\pi}: N \rightarrow N_{D}$ which maps the fan $\Sigma$ into $\Sigma_{D}$, such that $\pi^{*}[Y]=[D]$ for some ample divisor $Y$ on $\mathbf{P}_{\Sigma_{D}}$. Moreover, $\operatorname{dim} \mathbf{P}_{\Sigma_{D}}=i$, and the fan $\Sigma_{D}$ is the normal fan of $\Delta_{D}$ for a torus invariant $D$.

Remark 1.3. The fan $\Sigma_{D}$ lies in the space $\left(N_{D}\right)_{\mathbb{R}}:=N_{\mathbb{R}} / N_{\mathbb{R}}^{\prime}$, where $N^{\prime}=\{v \in N$ : $\left.\psi_{D}(-v)=-\psi_{D}(v)\right\}$ is a sublattice of $N$ and $\psi_{D}$ is the support function associated with $D$. Also, $\Delta_{D}$ lies in $\left(M_{D}\right)_{\mathbb{R}}$, where $M_{D}:=N^{\prime \perp} \cap M$ (for details see M2, Section 1]).

Remark 1.4. The map $\pi$ in the above theorem is well known and the variety $\mathbf{P}_{\Sigma_{D}}$ can be represented as $\operatorname{Proj}\left(\oplus_{k \geq 0} H^{0}\left(\mathbf{P}_{\Sigma}, \mathcal{O}_{\mathbf{P}_{\Sigma}}(k D)\right)\right)$. The condition on the map says that it is surjective with connected fibers. It would be interesting to see an analogue of the above theorem in a broader context.

We will need to use the following intersection properties of semiample divisors.

Lemma 1.5. If $D$ is an i-semiample divisor on a complete toric variety $\mathbf{P}_{\Sigma}$, then the intersection number $\left(D^{k} \cdot V(\tau)\right)>0$ for any $\tau \in \Sigma(d-k)$, such that $\tilde{\pi}(\tau)$ is contained in a cone of $\Sigma_{D}(i-k)$, and $\left(D^{k} \cdot V(\tau)\right)=0$ for all other $\tau \in \Sigma(i-k)$.

Proof. The arguments are the same as for Lemma 1.4 in M1.

Let $D$ be a semiample (torus invariant) divisor in degree $\beta \in A_{d-1}\left(\mathbf{P}_{\Sigma}\right)$ for a complete toric variety $\mathbf{P}_{\Sigma}$. By Theorem 1.2, we have the associated toric morphism $\pi: \mathbf{P}_{\Sigma} \rightarrow \mathbf{P}_{\Sigma_{D}}$. Let also $\sigma \in \Sigma_{X}$ be the smallest cone, containing the image of the cone $\tau \in \Sigma$, and $\sigma^{\prime} \in \Sigma(d-i+\operatorname{dim} \sigma)$ be such that $\tau \subset \sigma^{\prime}$ and $\tilde{\pi}\left(\sigma^{\prime}\right) \subset \sigma$. From M2. Section 1] it follows that there is a natural commutative diagram:

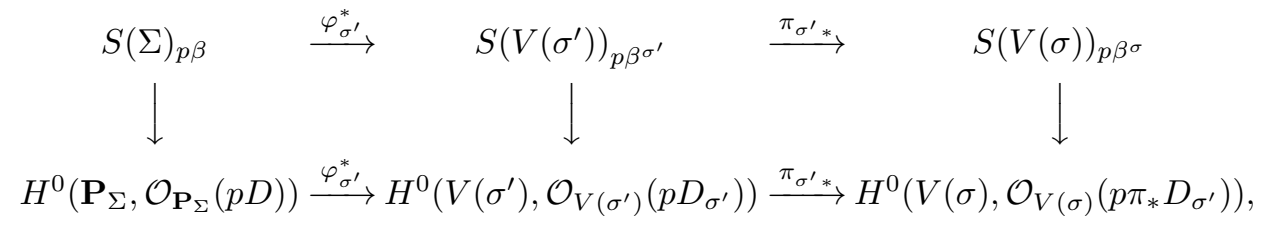

where $\beta^{\sigma^{\prime}}=\left[D_{\sigma^{\prime}}\right], D_{\sigma^{\prime}}:=\left.D\right|_{V\left(\sigma^{\prime}\right)}=D \cdot V\left(\sigma^{\prime}\right)$, in the Chow group of $V\left(\sigma^{\prime}\right)$, $\beta^{\sigma}=\pi_{*}\left[D_{\sigma^{\prime}}\right], \varphi_{\sigma^{\prime}}^{*}$ is the restriction of the global sections, the map $\pi_{\sigma^{\prime}}$ is a pushforward of the sections, and the vertical arrows are isomorphisms.

In this paper, we are interested in the nondegenerate hypersurfaces $X \subset \mathbf{P}_{\Sigma}$, which have only transversal intersections with the tori $\mathbf{T}_{\sigma}$. This is equivalent to saying that $x_{1} \partial f / \partial x_{1}, \ldots, x_{n} \partial f / \partial x_{n}$ do not vanish simultaneously on $\mathbf{P}_{\Sigma}$, where $f \in S_{\beta}$ determines the hypersurface $X$. A generic hypersurface in a given semiample degree is nondegenerate, by [D, Proposition 6.8]. We have the following property for such hypersurfaces.

Proposition 1.6. M2 Let $X$ be a semiample nondegenerate hypersurface in a complete toric variety $\mathbf{P}_{\Sigma}$, and let $\pi: \mathbf{P}_{\Sigma} \rightarrow \mathbf{P}_{\Sigma_{X}}$ be the associated morphism for $[X] \in A_{d-1}\left(\mathbf{P}_{\Sigma}\right)$, then $Y=\pi(X)$ is a nondegenerate ample hypersurface, and $X=\pi^{-1}(Y)$. 
For the affine hypersurfaces in tori, there is a Lefschetz-type theorem.

Theorem 1.7. Let $X$ be an i-semiample nondegenerate hypersurface in a complete toric variety $\mathbf{P}_{\Sigma}$. Then the natural restrictions

$$
H^{l}(\mathbf{T}) \rightarrow H^{l}(X \cap \mathbf{T})
$$

are isomorphisms for $l<i-1$, where $\mathbf{T} \subset \mathbf{P}_{\Sigma}$ is the maximal dimensional torus.

Proof. This follows from the corresponding result (see [DK]), when the hypersurface is ample, equation (2) in [M1] and the Künneth isomorphism.

In [M2, Section 5], we proved that the cohomology of a semiample nondegenerate hypersurface $X$ in a complete simplicial toric variety $\mathbf{P}_{\Sigma}$ has a direct sum decomposition:

$$
H^{*}(X)=H_{\text {toric }}^{*}(X) \oplus H_{\text {res }}^{*}(X),
$$

where $H_{\text {toric }}^{*}(X):=\operatorname{im}\left(H^{*}\left(\mathbf{P}_{\Sigma}\right) \stackrel{i^{*}}{\longrightarrow} H^{*}(X)\right)$ and $H_{\text {res }}^{*}(X):=\operatorname{im}\left(H^{*+1}\left(\mathbf{P}_{\Sigma} \backslash X\right) \stackrel{\text { Res }}{\longrightarrow}\right.$ $\left.H^{*}(X)\right)$ are called the toric and residue parts of the cohomology. These parts are orthogonal to each other:

$$
\int_{X} H_{\text {toric }}^{*}(X) \cup H_{\text {res }}^{*}(X)=0 .
$$

In [M2, Theorem 5.1], we also calculated

$$
H_{\text {toric }}^{*}(X) \cong H^{*}\left(\mathbf{P}_{\Sigma}\right) / \operatorname{Ann}([X])
$$

where $\operatorname{Ann}([X])$ is the annihilator of the class $[X] \in H^{2}\left(\mathbf{P}_{\Sigma}\right)$. The cohomology of $\mathbf{P}_{\Sigma}$ is isomorphic to

$$
\mathbb{C}\left[D_{1}, \ldots, D_{n}\right] /(P(\Sigma)+S R(\Sigma)),
$$

where the generators correspond to the torus invariant divisors of $\mathbf{P}_{\Sigma}$, and where

$$
\begin{gathered}
P(\Sigma)=\left\langle\sum_{i=1}^{n}\left\langle m, e_{i}\right\rangle D_{i}: m \in M\right\rangle, \\
S R(\Sigma)=\left\langle D_{i_{1}} \cdots D_{i_{k}}:\left\{e_{i_{1}}, \ldots, e_{i_{k}}\right\} \not \subset \sigma \text { for all } \sigma \in \Sigma\right\rangle .
\end{gathered}
$$

Hence, $H_{\text {toric }}^{*}(X)$ is isomorphic to the bigraded ring

$$
A_{1}(X)_{*, *}:=\mathbb{C}\left[D_{1}, \ldots, D_{n}\right] / I,
$$

where $I=(P(\Sigma)+S R(\Sigma)):[X]$ is the ideal quotient, and $D_{k}$ have the degree $(1,1)$.

We finish this section with some additional frequently used notation: $S(V(\tau))$, for $\tau \in \Sigma$, is the homogeneous coordinate ring of $V(\tau)$. $\varphi_{\tau}: X \cap V(\tau) \subset X$ is the inclusion for $\tau \in \Sigma$.

$\varphi_{\tau, \gamma}: X \cap V(\gamma) \subset X \cap V(\tau)$ is the inclusion for $\tau, \gamma \in \Sigma$ such that $\tau \subset \gamma$. $f_{\tau}=\varphi_{\tau}^{*}(f) \in S(V(\tau))$ denotes the polynomial defining $X \cap V(\tau)$ for $\tau \in \Sigma$.

$\operatorname{int} \tau=\tau \backslash\left(\bigcup_{\gamma \prec \tau} \gamma\right)$ is the relative interior of a cone $\tau$, where the union is by all proper faces of $\tau$.

$\pi_{\tau}: V(\tau) \rightarrow V(\sigma)$ is the restriction of $\pi$ for $\tau \in \Sigma$ and $\sigma \in \Sigma_{X}$ such that $\tilde{\pi}(\operatorname{int} \tau) \subset$ $\operatorname{int} \sigma$.

$f_{\sigma}=\pi_{\tau *}\left(f_{\tau}\right) \in S(V(\sigma))$ is the polynomial defining the hypersurface $\pi(X) \cap V(\sigma)$ in $V(\sigma)$.

$d(\tau):=d-\operatorname{dim} \tau$ for $\tau \in \Sigma$, and $i(\sigma):=i-\operatorname{dim} \sigma$ for $\sigma \in \Sigma_{X}$.

$\Gamma_{\sigma} \subset \Delta_{D}$ is the face corresponding to $\sigma \in \Sigma_{D}$.

$\beta_{1}^{\sigma}=\operatorname{deg}\left(\prod_{\tilde{\pi}\left(\rho_{k}\right) \subset \sigma} x_{k}\right) \in A_{d-1}\left(\mathbf{P}_{\Sigma}\right)$ for $\sigma \in \Sigma_{X}$. 


\section{The Hodge Structure of Semiample nondegenerate hyPersurfaces.}

The goal of this section is to compute the residue part of the cohomology of semiample nondegenerate hypersurfaces. This will completely generalize our previous results in [M1] and [M2], concerning the middle cohomology of big and nef hypersurfaces. The essential idea comes from the algorithmic approach in M2, Section 5] to computing of the residue part of cohomology. In fact, our proof will use an induction, where [M1, Theorem 4.4] may be considered as the base for this induction. This allows us to get an explicit description of the generators of cohomology. Quite remarkably, the Poincaré duality does the rest of the job: it gives all the relations among the generators.

Let $X$ be an $i$-semiample nondegenerate hypersurface in a complete simplicial toric variety $\mathbf{P}_{\Sigma}$, and let $\pi: \mathbf{P}_{\Sigma} \rightarrow \mathbf{P}_{\Sigma_{X}}$ be the associated contraction. From the diagram (14) in [M2], we have an exact sequence:

$$
\bigoplus_{k=1}^{n} H_{\mathrm{res}}^{s-2}\left(X \cap D_{k}\right) \stackrel{\oplus \varphi_{\rho_{k !} !}}{\longrightarrow} H_{\mathrm{res}}^{s}(X) \rightarrow \mathrm{Gr}_{s}^{W} P H^{s}(X \cap \mathbf{T}) \rightarrow 0 .
$$

A similar sequence can be written for $X \cap D_{k} \subset D_{k}$ instead of $X \subset \mathbf{P}_{\Sigma}$ as well as for all hypersurfaces $X \cap V(\tau)$ in the toric subvarieties $V(\tau)$ :

$$
\bigoplus_{\tau \subset \tau^{\prime} \in \Sigma(\operatorname{dim} \tau+1)} H_{\mathrm{res}}^{s-2}\left(X \cap V\left(\tau^{\prime}\right)\right) \rightarrow H_{\mathrm{res}}^{s}(X \cap V(\tau)) \rightarrow \operatorname{Gr}_{s}^{W} P H^{s}\left(X \cap \mathbf{T}_{\tau}\right) \rightarrow \underset{\text { (1) }}{0 .}
$$

Our first step is to show how to split the residue part $H_{\text {res }}^{s}(X \cap V(\tau))$. Then it will be clear how to obtain a description of $H_{\mathrm{res}}^{s}(X)$ from the above exact sequences. We closely follow the arguments in [M2, Section 6]. By the construction of the fan $\Sigma_{X}$ there is the smallest cone $\sigma \in \Sigma_{X}$, containing the image of the cone $\tau \in \Sigma$. The map $\pi$ restricted to the toric subvariety $V(\tau) \subset \mathbf{P}_{\Sigma}$ gives the contraction $\pi_{\tau}: V(\tau) \rightarrow V(\sigma)$. We will need to verify some information with a help of a simplicial toric subvariety $V\left(\sigma^{\prime}\right) \subset V(\tau)$ associated with a cone $\sigma^{\prime} \in \Sigma(d-i+\operatorname{dim} \sigma)$ such that $\tau \subset \sigma^{\prime}$ and the image of $\sigma^{\prime}$ is in $\sigma$. In this case, the toric variety $V\left(\sigma^{\prime}\right)$ is mapped birationally onto $V(\sigma)$, and we get a commutative diagram

$$
\begin{array}{cc}
H^{0}\left(V(\sigma), \Omega_{V(\sigma)}^{i(\sigma)}\left(* Y_{\sigma}\right)\right) \stackrel{\pi_{\tau}^{*} \longrightarrow}{\longrightarrow} & H^{0}\left(V(\tau), \Omega_{V(\tau)}^{i(\sigma)}\left(* X_{\tau}\right)\right) \\
\searrow_{\sigma^{\prime}}^{*} & \underset{\downarrow}{\mid \varphi_{\tau, \sigma^{\prime}}^{*}} \\
& H^{0}\left(V\left(\sigma^{\prime}\right), \Omega_{V\left(\sigma^{\prime}\right)}^{i(\sigma)}\left(* X_{\sigma^{\prime}}\right)\right),
\end{array}
$$

where we use short notations: $i(\sigma)=i-\operatorname{dim} \sigma, Y_{\sigma}=Y \cap V(\sigma), X_{\tau}=X \cap V(\tau)$, $X_{\sigma^{\prime}}=X \cap V\left(\sigma^{\prime}\right)$. There is a natural map

$$
S(V(\sigma))_{(p+1) \beta^{\sigma}-\beta_{0}^{\sigma}} \rightarrow H^{0}\left(V(\sigma), \Omega_{V(\sigma)}^{i(\sigma)}\left((p+1) Y_{\sigma}\right)\right)
$$

sending a polynomial $A$ to the form $A \Omega_{V(\sigma)} / f_{\sigma}^{p+1}$, where $\Omega_{V(\sigma)}$ is the $i(\sigma)$-form for the complete toric variety $V(\sigma)$, corresponding to a basis $m_{1}^{\sigma}, \ldots, m_{i(\sigma)}^{\sigma}$ of the lattice $M_{X} \cap \sigma^{\perp}$ (see [B, Definition 9.3]). The complete toric variety $V(\sigma)$ has homogeneous coordinates $y_{\gamma}$ corresponding to the integral generators $e_{\gamma}$ of the 1dimensional cones of the fan of $V(\sigma)$. We want to compute the pull-back of the form $A \Omega_{V(\sigma)} /\left(f_{\sigma}\right)^{p+1}$ with respect to the maps in (2). Since the form vanishes 
outside the torus $\mathbf{T}_{\sigma}$, the calculation can be done using the affine coordinates

$$
t_{j}^{\sigma}=\prod_{\gamma} y_{\gamma}^{\left\langle m_{j}^{\sigma}, e_{\gamma}\right\rangle}, \quad j=1, \ldots, i(\sigma),
$$

on the torus. Under the map $\pi_{\sigma^{\prime}}$, the coordinates $t_{j}^{\sigma}$ are identified with the mono-

mials $\prod_{\gamma^{\prime}} x_{\gamma^{\prime}}^{\left\langle m_{j}^{\sigma}, e_{\gamma^{\prime}}\right\rangle}$, where $x_{\gamma^{\prime}}$, for $\sigma^{\prime} \subset \gamma^{\prime} \in \Sigma\left(\operatorname{dim}\left(\sigma^{\prime}\right)+1\right)$, are the homogeneous coordinates on $V\left(\sigma^{\prime}\right)$, corresponding to the integral generators $e_{\gamma^{\prime}}$. Note

$$
\begin{aligned}
\Omega_{V(\sigma)}=\prod_{\gamma} y_{\gamma}\left(\sum_{\gamma}\left\langle m_{1}^{\sigma}, e_{\gamma}\right\rangle \frac{\mathrm{d} y_{\gamma}}{y_{\gamma}}\right) \wedge \cdots \wedge\left(\sum_{\gamma}\left\langle m_{i(\sigma)}^{\sigma}, e_{\gamma}\right\rangle \frac{\mathrm{d} y_{\gamma}}{y_{\gamma}}\right) \\
=\prod_{\gamma} y_{\gamma} \frac{\mathrm{d} t_{1}^{\sigma}}{t_{1}^{\sigma}} \wedge \cdots \wedge \frac{\mathrm{d} t_{i(\sigma)}^{\sigma}}{t_{i(\sigma)}^{\sigma}} .
\end{aligned}
$$

Therefore, we get

$$
\begin{gathered}
\pi_{\sigma^{\prime}}^{*}\left(\frac{A \Omega_{V(\sigma)}}{f_{\sigma}^{p+1}}\right)=\pi_{\sigma^{\prime}}^{*}\left(\frac{A \prod_{\gamma} y_{\gamma}}{f_{\sigma}^{p+1}}\right) \frac{\mathrm{d} t_{1}^{\sigma}}{t_{1}^{\sigma}} \wedge \cdots \wedge \frac{\mathrm{d} t_{i(\sigma)}^{\sigma}}{t_{i(\sigma)}^{\sigma}} \\
=\frac{\pi_{\sigma^{\prime}}^{*}\left(A \prod_{\gamma} y_{\gamma}\right)}{f_{\sigma^{\prime}}^{p+1}}\left(\sum_{\gamma^{\prime}}\left\langle m_{1}^{\sigma}, e_{\gamma^{\prime}}\right\rangle \frac{\mathrm{d} x_{\gamma^{\prime}}}{x_{\gamma^{\prime}}}\right) \wedge \cdots \wedge\left(\sum_{\gamma^{\prime}}\left\langle m_{i(\sigma)}^{\sigma}, e_{\gamma^{\prime}}\right\rangle \frac{\mathrm{d} x_{\gamma^{\prime}}}{x_{\gamma^{\prime}}}\right) \\
=\frac{\pi_{\sigma^{\prime}}^{*}\left(A \prod_{\gamma} y_{\gamma}\right)}{\left(\prod_{\gamma^{\prime}} x_{\gamma^{\prime}}\right) f_{\sigma^{\prime}}^{p+1} \Omega_{V\left(\sigma^{\prime}\right)},}
\end{gathered}
$$

where $\Omega_{V\left(\sigma^{\prime}\right)}$ is the $i(\sigma)$-form for the complete simplicial toric variety $V\left(\sigma^{\prime}\right)$, corresponding to the basis $m_{1}^{\sigma}, \ldots, m_{i(\sigma)}^{\sigma}$ of the lattice $M \cap \sigma^{\perp}=M_{X} \cap \sigma^{\perp}$ (see $\mathrm{BC}$, Definition 9.3]).

Similarly to the above, we can also compute the pull-back $\pi_{\tau}^{*}$ of the form $A \Omega_{V(\sigma)} / f_{\sigma}^{p+1}$. But now the coordinates $t_{j}^{\sigma}$ can be pulled by the map $\pi_{\tau}$ to the torus $\mathbf{T}_{\tau}$ and expressed in terms of the homogeneous coordinates of $\mathbf{P}_{\Sigma}$ :

$$
t_{j}^{\sigma}=\prod_{k=1}^{n} x_{k}^{\left\langle m_{j}^{\sigma}, e_{k}\right\rangle} .
$$

Completing $m_{1}^{\sigma}, \ldots, m_{i(\sigma)}^{\sigma}$ to a basis of the lattice $M \cap \tau^{\perp}$, we get a full set of coordinates $t_{1}^{\sigma}, \ldots, t_{i(\sigma)}^{\sigma}, t_{i(\sigma)+1}^{\sigma}, \ldots, t_{d-\operatorname{dim} \tau}^{\sigma}$ on the torus $\mathbf{T}_{\tau}$. Changing the basis, if necessary, we may also assume that these coordinates are well defined on the other tori which map onto $\mathbf{T}_{\sigma}$. On all such tori the map $\pi_{\tau}$ is given by projecting to the first $i(\sigma)$ affine coordinates. Consequently, we get

$$
\begin{array}{r}
\pi_{\tau}^{*}\left(\frac{A \Omega_{V(\sigma)}}{f_{\sigma}^{p+1}}\right)=\pi_{\tau}^{*}\left(\frac{A \prod_{\gamma} y_{\gamma}}{f_{\sigma}^{p+1}}\right) \frac{\mathrm{d} t_{1}^{\sigma}}{t_{1}^{\sigma}} \wedge \cdots \wedge \frac{\mathrm{d} t_{i(\sigma)}^{\sigma}}{t_{i(\sigma)}^{\sigma}} \\
=\frac{\pi_{\tau}^{*}\left(A \prod_{\gamma} y_{\gamma}\right)}{f^{p+1}}\left(\sum_{k=1}^{n}\left\langle m_{1}^{\sigma}, e_{k}\right\rangle \frac{\mathrm{d} x_{k}}{x_{k}}\right) \wedge \cdots \wedge\left(\sum_{k=1}^{n}\left\langle m_{i(\sigma)}^{\sigma}, e_{k}\right\rangle \frac{\mathrm{d} x_{k}}{x_{k}}\right) \\
=\frac{\pi_{\tau}^{*}\left(A \prod_{\gamma} y_{\gamma}\right)}{\left(\prod_{\tilde{\pi}\left(\rho_{k}\right) \not \subset \sigma} x_{k}\right) f^{p+1}} \Omega_{\sigma},
\end{array}
$$


where we introduce an $i(\sigma)$-form on the toric variety $V(\tau)$ :

$$
\Omega_{\sigma}:=\prod_{\tilde{\pi}\left(\rho_{k}\right) \not \subset \sigma} x_{k}\left(\sum_{k=1}^{n}\left\langle m_{1}^{\sigma}, e_{k}\right\rangle \frac{\mathrm{d} x_{k}}{x_{k}}\right) \wedge \cdots \wedge\left(\sum_{k=1}^{n}\left\langle m_{i(\sigma)}^{\sigma}, e_{k}\right\rangle \frac{\mathrm{d} x_{k}}{x_{k}}\right) .
$$

Here, we slightly abused the notation using the identification

$$
\left(S /\left\langle x_{k}: \tilde{\pi}\left(\rho_{k}\right) \subset \sigma\right\rangle\right)_{(p+1) \beta} \cong S(V(\tau))_{(p+1) \beta},
$$

which follows the same way as the equation (17) in [M2].

From the above calculations we can see a commutative diagram:

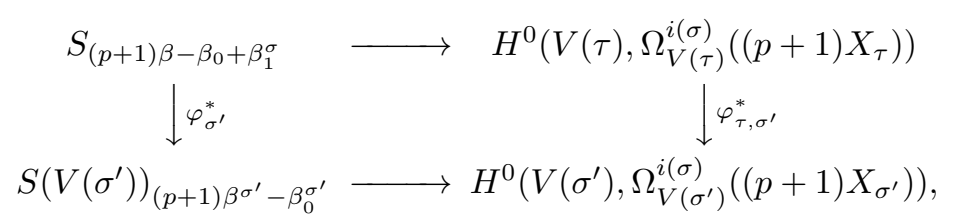

where $\beta_{1}^{\sigma}=\operatorname{deg}\left(\prod_{\tilde{\pi}\left(\rho_{k}\right) \subset \sigma} x_{k}\right)$ and the horizontal arrows are represented by the forms

$$
\frac{B \Omega_{\sigma}}{f^{p+1}} \text { and } \frac{B^{\prime} \Omega_{V\left(\sigma^{\prime}\right)}}{f_{\sigma^{\prime}}^{p+1}}
$$

for the corresponding polynomials $B$ and $B^{\prime}$, while the arrow on the left is defined by

$$
\varphi_{\sigma^{\prime}}^{*}(B):=\frac{\varphi_{\sigma^{\prime}}^{*}\left(B \prod_{\tilde{\pi}\left(\rho_{k}\right) \not \subset \sigma} x_{k}\right)}{\prod_{\gamma^{\prime}} x_{\gamma^{\prime}}} .
$$

Applying the residue map, we get another diagram:

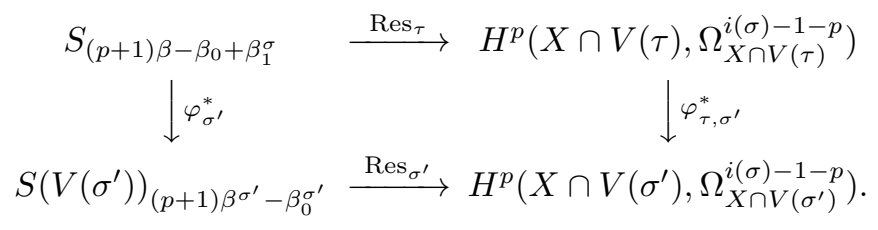

By [M1, Theorem 4.4], we know that the bottom arrow induces a well defined map

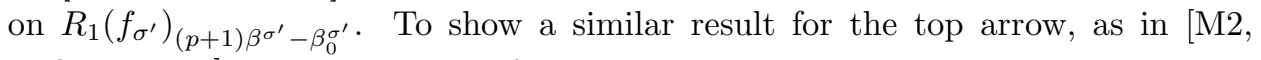
Definition 6.5], we introduce the following rings.

Definition 2.1. Given $f \in S_{\beta}$ of semiample degree $\beta=[D] \in A_{d-1}\left(\mathbf{P}_{\Sigma}\right)$ and $\sigma \in \Sigma_{D}$, let $J_{0}^{\sigma}(f)$ be the ideal in $S$ generated by the ideal $J_{0}(f)$ and all $x_{k}$ such that $\tilde{\pi}\left(\rho_{k}\right) \subset \sigma$, and let $J_{1}^{\sigma}(f)$ be the ideal quotient $J_{0}^{\sigma}(f):\left(\prod_{\tilde{\pi}\left(\rho_{k}\right) \not \subset \sigma} x_{k}\right)$. Then we get the quotient rings $R_{0}^{\sigma}(f)=S / J_{0}^{\sigma}(f)$ and $R_{1}^{\sigma}(f)=S / J_{1}^{\sigma}(f)$ graded by the Chow group $A_{d-1}\left(\mathbf{P}_{\Sigma}\right)$.

Proposition 2.2. Let $\beta=[D] \in A_{d-1}\left(\mathbf{P}_{\Sigma}\right)$ be $i$-semiample, and let $\sigma \in \Sigma_{D}$ and $\sigma^{\prime} \in \Sigma(d-i+\operatorname{dim} \sigma)$ be such that $\tilde{\pi}\left(\sigma^{\prime}\right) \subset \sigma$. If $\beta_{0}^{\sigma^{\prime}}=\operatorname{deg}\left(\prod_{\gamma^{\prime}} x_{\gamma^{\prime}}\right) \in A_{i(\sigma)-1}\left(V\left(\sigma^{\prime}\right)\right)$ and $\beta_{0}^{\sigma}=\operatorname{deg}\left(\prod_{\gamma} y_{\gamma}\right) \in A_{i(\sigma)-1}(V(\sigma))$ denote the anticanonical degrees, then, there are natural isomorphisms induced by $\varphi_{\sigma^{\prime}}^{*}$ and $\pi_{\sigma^{\prime} *}$ :

(i) $R_{0}^{\sigma}(f)_{* \beta} \cong R_{0}\left(f_{\sigma^{\prime}}\right)_{* \beta^{\sigma^{\prime}}} \cong R_{0}\left(f_{\sigma}\right)_{* \beta^{\sigma}}$

(ii) $R_{1}^{\sigma}(f)_{* \beta-\beta_{0}+\beta_{1}^{\sigma}} \cong R_{1}\left(f_{\sigma^{\prime}}\right)_{* \beta^{\sigma^{\prime}}-\beta_{0}^{\sigma^{\prime}}} \cong R_{1}\left(f_{\sigma}\right)_{* \beta^{\sigma}-\beta_{0}^{\sigma}}$.

(iii) $R_{0}^{\sigma}(f)_{* \beta-\beta_{0}+\beta_{1}^{\sigma}} \cong R_{0}\left(f_{\sigma^{\prime}}\right)_{* \beta^{\sigma^{\prime}}-\beta_{0}^{\sigma^{\prime}}} \cong R_{0}\left(f_{\sigma}\right)_{* \beta^{\sigma}-\beta_{0}^{\sigma}}$. 
Proof. This is just a generalization of Proposition 6.6 in $[\mathrm{M} 2$, and the proof follows the same way.

In order to prove that the top arrow in (3) is well defined on $R_{1}^{\sigma}(f)_{(p+1) \beta-\beta_{0}+\beta_{1}^{\sigma}}$ we will need to use an explicit representation of the $(i(\sigma)-1-p, p)$ Hodge component of the residue $\operatorname{Res}\left(B \Omega_{\sigma} / f^{p+1}\right) \in H^{i(\sigma)-1}(X \cap V(\tau))$.

Proposition 2.3. Let $X \subset \mathbf{P}_{\Sigma}$ be an $i$-semiample nondegenerate hypersurface defined by $f \in S_{\beta}$. Given $\tau \in \Sigma$ and the smallest cone $\sigma \in \Sigma_{X}$, containing the image of $\tau$, then, under the natural map

$$
\check{H}^{p}\left(\left.\mathcal{U}\right|_{X \cap V(\tau)}, \Omega_{X \cap V(\tau)}^{i(\sigma)-1-p}\right) \rightarrow H^{p}\left(X \cap V(\tau), \Omega_{X \cap V(\tau)}^{i(\sigma)-1-p}\right) \cong H^{i(\sigma)-1-p, p}(X \cap V(\tau)),
$$

the Hodge component of the residue $\operatorname{Res}_{\tau}\left(A \Omega_{\sigma} / f^{p+1}\right)=\operatorname{Res}\left(A \Omega_{\sigma} / f^{p+1}\right)^{i(\sigma)-1-p, p}$, for $A \in S_{(p+1) \beta-\beta_{0}+\beta_{1}^{\sigma}}$, is represented by the Cech cocycle

$$
\frac{(-1)^{i(\sigma)-1+(p(p+1) / 2)}}{p !}\left\{\frac{A K_{i_{p}} \cdots K_{i_{0}} \Omega_{\sigma}}{f_{i_{0}} \cdots f_{i_{p}}}\right\}_{i_{0} \ldots i_{p}} \in C^{p}\left(\left.\mathcal{U}\right|_{X \cap V(\tau)}, \Omega_{X \cap V(\tau)}^{i(\sigma)-1-p}\right),
$$

where $\mathcal{U}=\left\{U_{i}\right\}_{i=1}^{n}$ with $U_{i}=\left\{x \in \mathbf{P}_{\Sigma}: x_{i} f_{i}(x) \neq 0\right\}, f_{i}:=\partial f / \partial x_{i}$, is the open cover of $\mathbf{P}_{\Sigma}$, and where $K_{i}$ is the contraction operator $\left.\frac{\partial}{\partial x_{i}}\right\lrcorner$.

Proof. This proposition generalizes our previous results (see M1, Theorem 3.3] and [M2, Proposition 7.2]) as well as the corresponding results in [CaG]. However, here we suggest another way to do calculations. There is no need to plunge into hypercohomology as it was done in $\mathrm{CaG}$. Since we are interested only in the particular Hodge component of the residue, the two exact sequences

$$
\begin{aligned}
0 \rightarrow \Omega_{V(\tau)}^{i(\sigma)-p}\left(\log X_{\tau}\right) \rightarrow \Omega_{V(\tau)}^{i(\sigma)-p}\left(X_{\tau}\right) \stackrel{\mathrm{d}}{\rightarrow} \Omega_{V(\tau)}^{i(\sigma)-p+1}\left(2 X_{\tau}\right) / \Omega_{V(\tau)}^{i(\sigma)-p+1}\left(X_{\tau}\right) \stackrel{\mathrm{d}}{\rightarrow} \\
\quad \cdots \stackrel{\mathrm{d}}{\rightarrow} \Omega_{V(\tau)}^{d(\tau)}\left((d(\tau)-i(\sigma)+p+1) X_{\tau}\right) / \Omega_{V(\tau)}^{d(\tau)}\left((d(\tau)-i(\sigma)+p) X_{\tau}\right) \rightarrow 0
\end{aligned}
$$

(here, $d(\tau):=\operatorname{dim}(V(\tau))=d-\operatorname{dim} \tau$; see [BC, Proposition 10.1]) and

$$
0 \rightarrow \Omega_{V(\tau)}^{i(\sigma)-p} \rightarrow \Omega_{V(\tau)}^{i(\sigma)-p}\left(\log X_{\tau}\right) \stackrel{\text { Res }}{\longrightarrow} \Omega_{X_{\tau}}^{i(\sigma)-p-1} \rightarrow 0
$$

suffice to produce a representative of the component. Define auxiliary sheaves $L_{s}$ as the kernels of the morphisms

$\Omega_{V(\tau)}^{i(\sigma)-p+s-1}\left(s X_{\tau}\right) / \Omega_{V(\tau)}^{i(\sigma)-p+s-1}\left((s-1) X_{\tau}\right) \stackrel{\mathrm{d}}{\rightarrow} \Omega_{V(\tau)}^{i(\sigma)-p+s}\left((s+1) X_{\tau}\right) / \Omega_{V(\tau)}^{i(\sigma)-p+s}\left(s X_{\tau}\right)$ for $s=1, \ldots, p+1$. Then we get the short exact sequences

$$
0 \rightarrow L_{s} \rightarrow \Omega_{V(\tau)}^{i(\sigma)-p+s-1}\left(s X_{\tau}\right) / \Omega_{V(\tau)}^{i(\sigma)-p+s-1}\left((s-1) X_{\tau}\right) \rightarrow L_{s+1} \rightarrow 0
$$

giving rise to the maps in cohomology:

$$
\begin{aligned}
& H^{0}\left(V(\tau), L_{p+1}\right) \rightarrow H^{1}\left(V(\tau), L_{p}\right) \rightarrow \\
& \quad \cdots \rightarrow H^{p-1}\left(V(\tau), L_{2}\right) \rightarrow H^{p}\left(V(\tau), \Omega_{V(\tau)}^{i(\sigma)-p}\left(\log X_{\tau}\right)\right) .
\end{aligned}
$$

The first part of our proof is to show that the form $A \Omega_{\sigma} / f^{p+1}$ is a section in $H^{0}\left(V(\tau), L_{p+1}\right)$. For this it suffices to check that the differential of this form is a form on $V(\tau)$ with the order of the pole along $X_{\tau}$ at most $p+1$. Since

$$
\mathrm{d}\left(\frac{A \Omega_{\sigma}}{f^{p+1}}\right)=\frac{\mathrm{d}\left(A \Omega_{\sigma}\right)}{f^{p+1}}-\frac{(p+1) A \mathrm{~d} f \wedge \Omega_{\sigma}}{f^{p+2}}
$$


the desired property would follow from the fact:

$$
\mathrm{d} f \wedge \Omega_{\sigma} \equiv 0 \quad \text { modulo multiples of } f, x_{k} \text { and } \mathrm{d} x_{k} \text { for } \tilde{\pi}\left(\rho_{k}\right) \subset \tau \text {. }
$$

In order to prove this equation, note that $\Omega_{\sigma}$ is equal up to a multiple by a constant to

$$
\frac{x_{l_{1}} \cdots x_{l_{\operatorname{dim}\left(\sigma^{\prime}\right)}} K_{l_{\operatorname{dim}\left(\sigma^{\prime}\right)}} \cdots K_{l_{1}} \Omega}{\prod_{\tilde{\pi}\left(\rho_{k}\right) \subset \sigma} x_{k}}
$$

(where, $\Omega$ is the $d$-form for $\mathbf{P}_{\Sigma}$ from $[\mathrm{BQ}$, Definition 9.3]) for the integral generators $e_{l_{1}}, \ldots, e_{l_{\operatorname{dim}\left(\sigma^{\prime}\right)}}$ of a cone $\sigma^{\prime} \in \Sigma(d-i+\operatorname{dim} \sigma)$ such that $\tau \subset \sigma^{\prime}$ and the image of $\sigma^{\prime}$ is in $\sigma$. This can be seen from the definition of $\Omega$ and $\Omega_{\sigma}$, and the fact that the choice of a different basis in the definition of $\Omega$ affects only the sign of the form. Hence, $\mathrm{d} f \wedge \Omega_{\sigma}$ is equal up to a multiple by a constant to

$$
\begin{gathered}
\mathrm{d} f \wedge \frac{x_{l_{1}} \cdots x_{l_{\operatorname{dim}\left(\sigma^{\prime}\right)}} K_{l_{\operatorname{dim}\left(\sigma^{\prime}\right)}} \cdots K_{l_{1}} \Omega}{\prod_{\tilde{\pi}\left(\rho_{k}\right) \subset \sigma} x_{k}} \\
=(-1)^{\operatorname{dim}\left(\sigma^{\prime}\right)} x_{l_{1}} \cdots x_{l_{\operatorname{dim}\left(\sigma^{\prime}\right)}} \frac{K_{l_{\operatorname{dim}\left(\sigma^{\prime}\right)} \cdots K_{l_{1}}(\mathrm{~d} f \wedge \Omega)}^{\prod_{\tilde{\pi}\left(\rho_{k}\right) \subset \sigma} x_{k}}}{\quad+\sum_{r=1}^{\operatorname{dim}\left(\sigma^{\prime}\right)}(-1)^{r-1} f_{l_{r}} x_{l_{1}} \cdots x_{l_{\operatorname{dim}\left(\sigma^{\prime}\right)}} \frac{K_{l_{\operatorname{dim}\left(\sigma^{\prime}\right)}} \cdots \widehat{K_{l_{r}}} \cdots K_{l_{1}} \Omega}{\prod_{\tilde{\pi}\left(\rho_{k}\right) \subset \sigma} x_{k}} .} .
\end{gathered}
$$

The first summand is a multiple of $f$ by equation (3) in [M1], and we claim that the second is a multiple of $x_{k}$ or $\mathrm{d} x_{k}$ for $e_{k} \in \tau$ such that its image is in the relative interior of $\sigma$. Indeed, each summand in $\Omega$ is a multiple of $x_{k}$ or $\mathrm{d} x_{k}$, while the argument in the proof of Lemma 4.2 in [M2] shows that $x_{l_{r}} f_{l_{r}}$ is divisible by all $x_{k}$ for $\tilde{\pi}\left(e_{k}\right)$ in the relative interior of $\sigma$. Thus, equation (5) is proved.

In the second part, we need to find a representative in the Cech cohomology of the image of the form $A \Omega_{\sigma} / f^{p+1}$ by the composition of the maps in (4). The form $A \Omega_{\sigma} / f^{p+1}$ has a representation in $\check{H}^{0}\left(\left.\mathcal{U}\right|_{V(\tau)}, L_{p+1}\right)$ by the Cech cocycle

$$
\left\{\frac{A \mathrm{~d} f \wedge K_{i_{0}} \Omega_{\sigma}}{f_{i_{0}} f^{p+1}}\right\}_{i_{0}}
$$

since $\mathrm{d} f \wedge K_{i_{0}} \Omega_{\sigma}=f_{i_{0}} \Omega_{\sigma}-K_{i_{0}}\left(\mathrm{~d} f \wedge \Omega_{\sigma}\right)$ and because of equation (5). Note that the maps between cohomologies in (4) are the connecting homomorphisms of long exact sequences, so that the image of the Čech cocycle representing $A \Omega_{\sigma} / f^{p+1}$ can be computed using the following commutative diagrams:

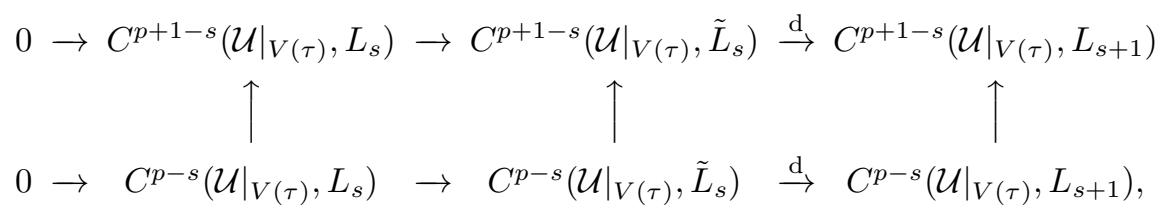

where $\tilde{L}_{s}:=\Omega_{V(\tau)}^{i(\sigma)-p+s-1}\left(s X_{\tau}\right) / \Omega_{V(\tau)}^{i(\sigma)-p+s-1}\left((s-1) X_{\tau}\right)$ and the vertical arrows are the Čech coboundary maps. When $s=p$, the cocycle representing $A \Omega_{\sigma} / f^{p+1}$ has a lift to the cochain

$$
-\frac{1}{p}\left\{\frac{A K_{i_{0}} \Omega_{\sigma}}{f_{i_{0}} f^{p}}\right\}_{i_{0}} \in C^{0}\left(\left.\mathcal{U}\right|_{V(\tau)}, \Omega_{V(\tau)}^{i(\sigma)-1}\left(p X_{\tau}\right) / \Omega_{V(\tau)}^{i(\sigma)-1}\left((p-1) X_{\tau}\right)\right),
$$


because

$$
\mathrm{d}\left(\frac{A K_{i_{0}} \Omega_{\sigma}}{f_{i_{0}} f^{p}}\right)=-p A \frac{\mathrm{d} f \wedge K_{i_{0}} \Omega_{\sigma}}{f_{i_{0}} f^{p+1}}+\frac{\mathrm{d}\left(A K_{i_{0}} \Omega_{\sigma}\right)}{f_{i_{0}} f^{p}}-\frac{A \mathrm{~d} f_{i_{0}} \wedge K_{i_{0}} \Omega_{\sigma}}{f^{p} f_{i_{0}}^{2}} .
$$

The Čech coboundary of this cochain is

$$
\begin{gathered}
-\frac{1}{p}\left\{\frac{A K_{i_{1}} \Omega_{\sigma}}{f_{i_{1}} f^{p}}-\frac{A K_{i_{0}} \Omega_{\sigma}}{f_{i_{0}} f^{p}}\right\}_{i_{0} i_{1}}=-\frac{1}{p}\left\{\frac{A\left(f_{i_{0}} K_{i_{1}} \Omega_{\sigma}-f_{i_{1}} K_{i_{0}} \Omega_{\sigma}\right)}{f_{i_{0}} f_{i_{1}} f^{p}}\right\}_{i_{0} i_{1}} \\
=-\frac{1}{p}\left\{\frac{A\left(-\mathrm{d} f \wedge K_{i_{1}} K_{i_{0}} \Omega_{\sigma}+K_{i_{1}} K_{i_{0}}\left(\mathrm{~d} f \wedge \Omega_{\sigma}\right)\right)}{f_{i_{0}} f_{i_{1}} f^{p}}\right\}_{i_{0} i_{1}} \\
=\frac{1}{p}\left\{\frac{A \mathrm{~d} f \wedge K_{i_{1}} K_{i_{0}} \Omega_{\sigma}}{f_{i_{0}} f_{i_{1}} f^{p}}\right\}_{i_{0} i_{1}},
\end{gathered}
$$

and, by the above diagram, it is a Čech cocycle in $C^{1}\left(\left.\mathcal{U}\right|_{V(\tau)}, L_{p}\right)$. Continuing this way, one gets the cocycle

$$
\begin{aligned}
& \frac{(-1)^{p}(-1)^{1+\cdots+p}}{p !}\left\{\frac{\operatorname{Ad} f \wedge K_{i_{p}} \cdots K_{i_{0}} \Omega_{\sigma}}{f_{i_{0}} \cdots f_{i_{p}} f}\right\}_{i_{0} \cdots i_{p}} \\
& =\frac{(-1)^{i(\sigma)-1+(p(p+1) / 2)}}{p !}\left\{\frac{A K_{i_{p}} \cdots K_{i_{0}} \Omega_{\sigma}}{f_{i_{0}} \cdots f_{i_{p}}} \wedge \frac{\mathrm{d} f}{f}\right\}_{i_{0} \ldots i_{p}}
\end{aligned}
$$

in $C^{p}\left(\left.\mathcal{U}\right|_{V(\tau)}, \Omega_{V(\tau)}^{i(\sigma)-p}\left(\log X_{\tau}\right)\right)$, which is ready for application of the residue map. This produces the desired result.

To compute the cup product on the residue part of cohomology of semiample hypersurfaces, we will need to use certain toric residue maps similar to $\bar{B}$ and M1. Let us first recall some facts about toric residues, and then apply them to our situation.

Definition 2.4. [C] Assume $F_{0}, \ldots, F_{d} \in S_{\beta}$ do not vanish simultaneously on a complete $d$-dimensional toric variety $\mathbf{P}$. Then the toric residue map

$$
\operatorname{Res}_{F}: S_{\rho} /\left\langle F_{0}, \ldots, F_{d}\right\rangle_{\rho} \rightarrow \mathbb{C}
$$

$\rho=(d+1) \beta-\beta_{0}$, is given by the formula $\operatorname{Res}_{F}(H)=\operatorname{Tr}_{\mathbf{P}}\left(\left[\varphi_{F}(H)\right]\right)$, where $\operatorname{Tr}_{\mathbf{P}}$ : $H^{d}\left(\mathbf{P}, \Omega_{\mathbf{P}}^{d}\right) \rightarrow \mathbb{C}$ is the trace map, and $\left[\varphi_{F}(H)\right]$ is the class represented by the $d$-form $H \Omega /\left(F_{0} \cdots F_{d}\right)$ in the Cech cohomology with respect to the open cover $\left\{x \in \mathbf{P}: F_{j}(x) \neq 0\right\}$.

In [M1, Definition 3.4] we introduced the following constant.

Definition 2.5. [M1] For $\beta=\left[\sum_{k=1}^{n} b_{k} D_{k}\right] \in A_{d-1}(\mathbf{P})$ and an ordered subset $I=$ $\left\{i_{0}, \ldots, i_{d}\right\}$ of $\{1, \ldots, n\}$, let the constant $c_{I}^{\beta}$ be the determinant of the $(d+1) \times(d+1)$ matrix obtained from $\left(\left\langle m_{j}, e_{k}\right\rangle_{1 \leq j \leq d, k \in I}\right)$ by adding the first row $\left(b_{k}\right)_{k \in I}$, where $m_{1}, \ldots, m_{d}$ is the same integer basis of the lattice $M$ as in the definition of $\Omega$.

The next result from [M1, Theorem 4.8] shows how to explicitly compute the toric residue map.

Theorem 2.6. M1 Let $\mathbf{P}$ be a complete toric variety, and let $\beta=[D] \in A_{d-1}(\mathbf{P})$ be big and nef. If $F_{0}, \ldots, F_{d} \in S_{\beta}$ do not vanish simultaneously on $\mathbf{P}$, then:

(i) the toric residue $\operatorname{map}_{\operatorname{Res}_{F}}: S_{\rho} /\left\langle F_{0}, \ldots, F_{d}\right\rangle_{\rho} \rightarrow \mathbb{C}, \rho=(d+1) \beta-\beta_{0}$, is an isomorphism, 
(ii) $\operatorname{Res}_{F}\left(J_{F}\right)=d ! \operatorname{vol}\left(\Delta_{D}\right)$, where $J_{F}=\operatorname{det}\left(\partial F_{j} / \partial x_{i_{k}}\right) / c_{I}^{\beta} \hat{x}_{I} \in S_{(d+1) \beta-\beta_{0}}$ is the toric Jacobian of $F_{0}, \ldots, F_{d}$, and $c_{I}^{\beta} \neq 0$.

When $X \subset \mathbf{P}$ is a nondegenerate hypersurface determined by $f \in S_{\beta}$, by Lemma 4.10 in [M1], the polynomials $F_{k}=x_{k} \partial f / \partial x_{k}, k \in I$, do not vanish simultaneously on $\mathbf{P}$ for $c_{I}^{\beta} \neq 0$, and $J_{0}(f)=\left\langle x_{k} \partial f / \partial x_{k}: k \in I\right\rangle$. Therefore, there is the corresponding toric residue map $\operatorname{Res}_{F_{I}}: S_{\rho} / J_{0}(f)_{\rho} \rightarrow \mathbb{C}$. However, the diagram (10) in M1] tells us that this map depends on the choice of $I$, while $c_{I}^{\beta} \operatorname{Res}_{F_{I}}$ doesn't. By the above theorem, $c_{I}^{\beta} \operatorname{Res}_{F_{I}}(J)=d ! \operatorname{vol}\left(\Delta_{D}\right)$ for

$$
J=\frac{\operatorname{det}\left(\partial F_{j} / \partial x_{k}\right)_{k, j \in I}}{\left(c_{I}^{\beta}\right)^{2} \hat{x}_{I}}
$$

For an $i$-semiample nondegenerate hypersurface $X \subset \mathbf{P}_{\Sigma}$ and $\sigma \in \Sigma_{X}$. By Proposition 2.2, there is an isomorphism

$$
R_{0}^{\sigma}(f)_{(i-\operatorname{dim} \sigma+1) \beta-\beta_{0}+\beta_{1}^{\sigma}} \cong R_{0}\left(f_{\sigma^{\prime}}\right)_{(i-\operatorname{dim} \sigma+1) \beta^{\sigma^{\prime}}-\beta_{0}^{\sigma^{\prime}}} \cong R_{0}\left(f_{\sigma}\right)_{(i-\operatorname{dim} \sigma+1) \beta^{\sigma}-\beta_{0}^{\sigma}},
$$

composition of which we denote by $\pi_{\sigma *}$. Since $Y \cap V(\sigma)$ is an ample hypersurface in $V(\sigma)$, determined by $f_{\sigma}$, we get the corresponding map

$$
c_{I}^{\beta^{\sigma}} \operatorname{Res}_{F_{\sigma I}}: R_{0}\left(f_{\sigma}\right)_{(i-\operatorname{dim} \sigma+1) \beta^{\sigma}-\beta_{0}^{\sigma}} \cong \mathbb{C} \text {. }
$$

It is not difficult to find a polynomial in $R_{0}\left(f_{\sigma}\right)_{(i-\operatorname{dim} \sigma+1) \beta^{\sigma}-\beta_{0}^{\sigma}}$, which $\varphi_{\sigma^{\prime} *}$ and $\pi_{\sigma *}$ map to the polynomials (6) associated with $f_{\sigma^{\prime}} \in S\left(V\left(\sigma^{\prime}\right)\right)_{\beta^{\sigma^{\prime}}}$ and $f_{\sigma} \in S(V(\sigma))_{\beta^{\sigma}}$, respectively. This polynomial is

$$
J_{\sigma}=\frac{\operatorname{det}\left(x_{k} \partial F_{j} / \partial x_{k}\right)_{k, j \in I}}{\left(c_{I}^{\beta, \sigma}\right)^{2} \prod_{\tilde{\pi}\left(\rho_{k}\right) \not \subset \sigma} x_{k}}
$$

where $c_{I}^{\beta, \sigma} \neq 0$ for an ordered subset $I$ of $\{1, \ldots, n\}$ is the determinant of the $(i(\sigma)+1) \times(i(\sigma)+1)$ matrix obtained from $\left(\left\langle m_{j}^{\sigma}, e_{k}\right\rangle\right)_{1 \leq j \leq i(\sigma), k \in I}$ by adding the first row $\left(b_{i}\right)_{i \in I}$ such that $\beta=\left[\sum_{k=1}^{n} b_{k} D_{k}\right]$ with $b_{k}=0$ if $\rho_{k} \subset \sigma$, and $m_{1}^{\sigma}, \ldots, m_{i(\sigma)}^{\sigma}$ is the same integer basis of the lattice $M_{X} \cap \sigma^{\perp}$ as in the definition of $\Omega_{\sigma}$. One can verify that $J_{\sigma}$ does not depend on the choice of $I$, and the fact that $J_{\sigma}$ is indeed a polynomial follows from the arguments at the end of the proof of $[\mathrm{C}$, Proposition 4.1]. Using Theorem 2.6, we conclude that

$$
c_{I}^{\beta^{\sigma}} \operatorname{Res}_{F_{\sigma}} \pi_{\sigma *}\left(J_{\sigma}\right)=(i-\operatorname{dim} \sigma) ! \operatorname{vol}\left(\Gamma_{\sigma}\right),
$$

where $\Gamma_{\sigma}$ is the face of $\Delta_{D}$ corresponding to $\sigma \in \Sigma_{X}$.

In the case $\operatorname{dim} \sigma=i-1$, we will also need to use a special map constructed the following way. By equation (7),

$$
C \prod_{\tilde{\pi}\left(\rho_{k}\right) \not \subset \sigma} x_{k}-\frac{c_{I}^{\beta^{\sigma}} \operatorname{Res}_{F_{\sigma}} \pi_{\sigma *}\left(C \prod_{\tilde{\pi}\left(\rho_{k}\right) \not \subset \sigma} x_{k}\right)}{\operatorname{vol}\left(\Gamma_{\sigma}\right)} J_{\sigma} \in J_{0}^{\sigma}(f)_{2 \beta-\beta_{0}+\beta_{1}^{\sigma}}
$$

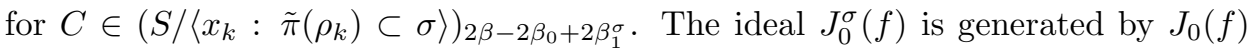
and $x_{k}$ for $\tilde{\pi}\left(\rho_{k}\right) \subset \sigma$, while the ideal $J_{0}(f)$ has two minimal generators $f$ and $x_{s} f_{s}$ if $\tilde{\pi}\left(\rho_{s}\right) \not \subset \sigma$. Define a projection $p_{\sigma}$ as the composition of the maps:

$$
\begin{aligned}
& \left.\left(S /\left\langle x_{k}: \tilde{\pi}\left(\rho_{k}\right) \subset \sigma\right\rangle\right)_{2 \beta-2 \beta_{0}+2 \beta_{1}^{\sigma}} \rightarrow J_{0}^{\sigma}(f)_{\rho} \rightarrow J_{0}^{\sigma}(f)_{\rho} /\left\langle f, x_{k}: \tilde{\pi}\left(\rho_{k}\right) \subset \sigma\right\rangle\right)_{\rho} \\
\cong & \left(\left\langle x_{s} f_{s}\right\rangle /\left\langle x_{k}: \tilde{\pi}\left(\rho_{k}\right) \subset \sigma\right\rangle\right)_{\rho} \cong\left(S /\left\langle x_{k}: \tilde{\pi}\left(\rho_{k}\right) \subset \sigma\right\rangle\right)_{\beta-\beta_{0}+\beta_{1}^{\sigma}}=R_{1}^{\sigma}(f)_{\beta-\beta_{0}+\beta_{1}^{\sigma}},
\end{aligned}
$$


where $\rho=2 \beta-\beta_{0}+\beta_{1}^{\sigma}$, the first map is defined by (8) and the fourth map is a division by $x_{s} f_{s} /\left\langle m_{1}^{\sigma}, e_{s}\right\rangle$. The map $p_{\sigma}$ is independent of the choice of $s$. Indeed, since

$$
\frac{e_{s_{1}}}{\left\langle m_{1}^{\sigma}, e_{s_{1}}\right\rangle}-\frac{e_{s_{2}}}{\left\langle m_{1}^{\sigma}, e_{s_{2}}\right\rangle}
$$

belongs to the space spanned by $e_{k}$ for $\tilde{\pi}\left(\rho_{k}\right) \subset \sigma$, there is an Euler formula (see [BC, Definition 3.9]) showing that

$$
\frac{x_{s_{1}} f_{s_{1}}}{\left\langle m_{1}^{\sigma}, e_{s_{1}}\right\rangle}-\frac{x_{s_{2}} f_{s_{2}}}{\left\langle m_{1}^{\sigma}, e_{s_{2}}\right\rangle}
$$

belongs to the ideal $\left\langle f, x_{k}: \tilde{\pi}\left(\rho_{k}\right) \subset \sigma\right\rangle$.

For $\sigma \in \Sigma_{X}$, define $\operatorname{Res}^{\sigma}: R_{1}^{\sigma}(f) \rightarrow \mathbb{C}$ by 0 in all degrees except $\left.i(\sigma)+1\right) \beta-$ $2 \beta_{0}+2 \beta_{1}^{\sigma}$, for which $\operatorname{Res}^{\sigma}$ is the composition

$$
R_{1}^{\sigma}(f)_{(i(\sigma)+1) \beta-2 \beta_{0}+2 \beta_{1}^{\sigma}} \rightarrow R_{0}^{\sigma}(f)_{(i(\sigma)+1) \beta-\beta_{0}+\beta_{1}^{\sigma}} \stackrel{c_{I}^{\beta^{\sigma}} \operatorname{Res}_{F_{\sigma I}} \pi_{\sigma *}}{\longrightarrow} \mathbb{C},
$$

where the first arrow is a multiplication by $\prod_{\tilde{\pi}\left(\rho_{k}\right) \not \subset \sigma} x_{k} /\left((i-\operatorname{dim} \sigma) ! \operatorname{vol}\left(\Gamma_{\sigma}\right)\right)$.

Now, we are ready to show how to partially split the residue part $H_{\mathrm{res}}^{s}(X \cap V(\tau))$.

Proposition 2.7. Let $X \subset \mathbf{P}_{\Sigma}$ be an i-semiample nondegenerate hypersurface defined by $f \in S_{\beta}$. Given $\tau \in \Sigma$ and the smallest cone $\sigma \in \Sigma_{X}$, containing the image of $\tau$, then: (i) for $p+q \neq i-\operatorname{dim} \sigma-1$,

$$
H_{\mathrm{res}}^{p, q}(X \cap V(\tau))=\sum_{\tau \subset \tau^{\prime} \in \Sigma(\operatorname{dim} \tau+1)} \varphi_{\tau, \tau^{\prime} !} H_{\mathrm{res}}^{p-1, q-1}\left(X \cap V\left(\tau^{\prime}\right)\right),
$$

and

$$
H_{\mathrm{res}}^{p, q}(X \cap V(\tau)) \cong R_{1}^{\sigma}(f)_{(q+1) \beta-\beta_{0}+\beta_{1}^{\sigma}} \bigoplus \sum_{\substack{\tau \subset \tau^{\prime} \\ \tau^{\prime} \in \Sigma(\operatorname{dim} \tau+1)}} \varphi_{\tau, \tau^{\prime} !} H_{\mathrm{res}}^{p-1, q-1}\left(X \cap V\left(\tau^{\prime}\right)\right)
$$

for $p+q=i-\operatorname{dim} \sigma-1$, where the first factor is included by the residue map,

(ii) for $\operatorname{dim} \sigma<i-1, A \in R_{1}^{\sigma}(f)_{(p+1) \beta-\beta_{0}+\beta_{1}^{\sigma}}, B \in R_{1}^{\sigma}(f)_{(q+1) \beta-\beta_{0}+\beta_{1}^{\sigma}}$,

$$
\operatorname{Res}_{\tau}(A) \cup \operatorname{Res}_{\tau}(B)=c_{p}^{\sigma} \operatorname{Res}^{\sigma}(A B) \varphi_{\tau}^{*} i^{*} X^{i(\sigma)-1},
$$

where

$$
c_{p}^{\sigma}=-(-2 \pi \sqrt{-1})^{i(\sigma)-1} \frac{(-1)^{(i(\sigma)-1)(i(\sigma)+2 p+2)}}{p !(i(\sigma)-p-1) !},
$$

if $p+q=i-\operatorname{dim} \sigma-1$, and

$$
\operatorname{Res}_{\tau}(A) \cup \operatorname{Res}_{\tau}(B)=0
$$

if $p+q \neq i-\operatorname{dim} \sigma-1$, and, for $\operatorname{dim} \sigma=i-1$,

$$
\operatorname{Res}_{\tau}(A) \cup \operatorname{Res}_{\tau}(B)=-\operatorname{Res}^{\sigma}(A B) \varphi_{\tau}^{*} i^{*}\left[\mathbf{P}_{\Sigma}\right]+\operatorname{Res}_{\tau}\left(p_{\sigma}(A B)\right) .
$$

Proof. (i) From the exact sequence (11) and the vanishing

$$
\operatorname{Gr}_{s}^{W} H^{s}\left(X \cap \mathbf{T}_{\tau}\right) \cong \operatorname{Gr}_{s}^{W} H^{s}\left(\left(Y \cap \mathbf{T}_{\sigma}\right) \times\left(\mathbb{C}^{*}\right)^{d-i+\operatorname{dim} \sigma-\operatorname{dim} \tau}\right)=0
$$

for $s \neq i-\operatorname{dim} \sigma-1$, by equations (2) and (11) in [M2], we conclude the first part of the statement.

For the second part, we first use an induction on the dimension of $\tau \in \Sigma$ such that $\sigma \in \Sigma_{X}$ is the smallest cone containing the image of $\tau$ to show that

$$
\operatorname{Res}_{\tau}\left(J_{1}^{\sigma}(f)_{(q+1) \beta-\beta_{0}+\beta_{1}^{\sigma}}\right)=0
$$


in the cohomology of $X \cap V(\tau)$. By Proposition 2.2 and [M2, Theorem 4.4], this is true for $\tau \in \Sigma(d-i+\operatorname{dim} \sigma)$ such that $\tilde{\pi}(\tau) \subset \sigma$, because $X \cap V(\tau)$ in $V(\tau)$ is big and nef. By induction, assume that (9) holds for $\tau^{\prime} \in \Sigma(k)$ such that $\sigma \in \Sigma_{X}$ is the smallest cone containing $\tilde{\pi}\left(\tau^{\prime}\right)$. We need to prove (9) for $\tau \in \Sigma(k-1)$ satisfying the same condition. Let $A \in J_{1}^{\sigma}(f)_{(q+1) \beta-\beta_{0}+\beta_{1}^{\sigma}}$, then, by Poincaré duality, $\operatorname{Res}_{\tau}(A)=0$ in $H^{p, q}(X \cap V(\tau)), p=i-\operatorname{dim} \sigma-1-q$, if and only if $\int_{X}$ of the cup product of $\operatorname{Res}_{\tau}(A)$ with all elements in $H^{d(\tau)-1-p, d(\tau)-1-q}(X \cap V(\tau))$ vanishes (note that $X$ is not necessarily connected). From Section 11 we know that

$$
H^{*}(X \cap V(\tau))=H_{\text {toric }}^{*}(X \cap V(\tau)) \oplus H_{\text {res }}^{*}(X \cap V(\tau)),
$$

and

$$
\int_{X} H_{\text {toric }}^{*}(X \cap V(\tau)) \cup H_{\text {res }}^{*}(X \cap V(\tau))=0 .
$$

On the other hand, for $\operatorname{dim} \tau \neq d-i+\operatorname{dim} \sigma$,

$H_{\mathrm{res}}^{d(\tau)-1-p, d(\tau)-1-q}(X \cap V(\tau))=\sum_{\tau \subset \tau^{\prime} \in \Sigma(\operatorname{dim} \tau+1)} \varphi_{\tau, \tau^{\prime} !} H_{\mathrm{res}}^{d(\tau)-2-p, d(\tau)-2-q}\left(X \cap V\left(\tau^{\prime}\right)\right)$

by the first part of this proposition. Since

$$
\operatorname{Res}_{\tau}(A) \cup \varphi_{\tau, \tau^{\prime} !} h=\varphi_{\tau, \tau^{\prime} !}\left(\varphi_{\tau, \tau^{\prime}}^{*} \operatorname{Res}_{\tau}(A) \cup h\right)
$$

by the Gysin projection formula, it is enough to show $\varphi_{\tau, \tau^{\prime}}^{*}\left(\operatorname{Res}_{\tau}(A)\right)=0$ for $\tau \subset \tau^{\prime} \in \Sigma(\operatorname{dim} \tau+1)$. If $\tilde{\pi}\left(\tau^{\prime}\right) \not \subset \sigma$, then there is $\rho_{l} \subset \tau^{\prime}$ such that $\tilde{\pi}\left(\rho_{l}\right) \not \subset \sigma$. In this case, by Proposition 2.3, $\varphi_{\tau, \tau^{\prime}}^{*} \operatorname{Res}_{\tau}(A)$ is represented by the restriction of the Čech cocycle

$$
\frac{(-1)^{i(\sigma)-1+(q(q+1) / 2)}}{q !}\left\{\frac{A K_{i_{q}} \cdots K_{i_{0}} \Omega_{\sigma}}{f_{i_{0}} \cdots f_{i_{q}}}\right\}_{i_{0} \ldots i_{q}}
$$

to $X \cap V\left(\tau^{\prime}\right)$. But each term in the form $K_{i_{q}} \cdots K_{i_{0}} \Omega_{\sigma}$ on a nonempty open set $U_{i_{q}} \cap \cdots \cap U_{i_{0}} \cap V\left(\tau^{\prime}\right)$ contains $\mathrm{d} x_{l}$ or $x_{l}$ and, therefore, vanishes on $X \cap V\left(\tau^{\prime}\right)$. If $\tilde{\pi}\left(\tau^{\prime}\right) \subset \sigma$, then, by the induction assumption, $\operatorname{Res}_{\tau^{\prime}}(A)=0$ in $H^{p, q}\left(X \cap V\left(\tau^{\prime}\right)\right)$. The commutative diagram

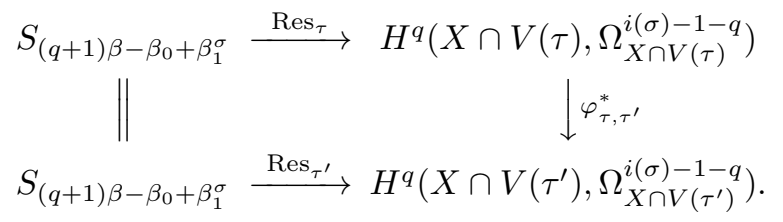

gives $\varphi_{\tau, \tau^{\prime}}^{*} \operatorname{Res}_{\tau}(A)=0$ again. This finishes the proof of (9) by induction, which shows that the map

$$
R_{1}^{\sigma}(f)_{(q+1) \beta-\beta_{0}+\beta_{1}^{\sigma}} \stackrel{\operatorname{Res}_{\tau}}{\longrightarrow} H_{\mathrm{res}}^{p, q}(X \cap V(\tau))
$$

is well defined.

Finally, for $\sigma^{\prime} \in \Sigma(d-i+\operatorname{dim} \sigma)$ such that $\tilde{\pi}\left(\sigma^{\prime}\right) \subset \sigma$, we have a diagram

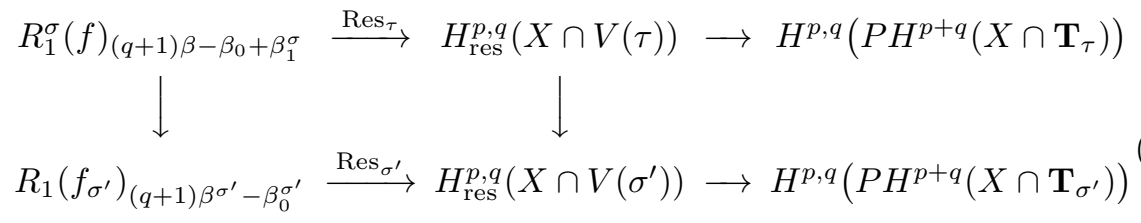


and, by equation (12) in [M2], the isomorphisms

$$
H^{p, q}\left(P H^{p+q}\left(X \cap \mathbf{T}_{\tau}\right)\right) \cong H^{p, q}\left(P H^{p+q}\left(\pi(X) \cap \mathbf{T}_{\sigma}\right)\right) \cong H^{p, q}\left(P H^{p+q}\left(X \cap \mathbf{T}_{\sigma^{\prime}}\right)\right)
$$

induced by $\pi: \mathbf{P}_{\Sigma} \rightarrow \mathbf{P}_{\Sigma_{X}}$. Since $X \cap V\left(\sigma^{\prime}\right)$ in $V\left(\sigma^{\prime}\right)$ is big and nef, the composition on the bottom of (11) is an isomorphism, by Theorem 4.4 in [M1. Hence, the same holds for the composition on the top of (11). Now, the exact sequence (11) produces the result.

(ii) Let $\operatorname{dim} \sigma<i-1$. As in part (i), we prove the formula

$$
\operatorname{Res}_{\tau}(A) \cup \operatorname{Res}_{\tau}(B)=c_{p}^{\sigma} \operatorname{Res}^{\sigma}(A B) \varphi_{\tau}^{*} i^{*} X^{i(\sigma)-1}
$$

by an induction on the dimension of $\tau \in \Sigma$ such that $\sigma \in \Sigma_{X}$ is the smallest cone containing the image of $\tau$. When $\operatorname{dim} \tau=d-i+\operatorname{dim} \sigma$, the hypersurface $X \cap V(\tau)$ in $V(\tau)$ is big and nef. Therefore, by the results in [M1, page 104] and the definition of $\operatorname{Res}^{\sigma}$,

$$
\int_{X \cap V(\tau)} \operatorname{Res}_{\tau}(A) \cup \operatorname{Res}_{\tau}(B)=c_{p}^{\sigma}(i-\operatorname{dim} \sigma) ! \operatorname{vol}\left(\Gamma_{\sigma}\right) \operatorname{Res}^{\sigma}(A B)
$$

(the formula we use in [M1, page 104] must be corrected by a factor of $2 \pi \sqrt{-1}$ ). By 昷, Section 5.3], $(X \cap V(\tau))^{i(\sigma)}=(i-\operatorname{dim} \sigma) ! \operatorname{vol}\left(\Gamma_{\sigma}\right)$, whence (12) holds in this case. Assume that (12) holds for $\operatorname{dim} \tau=k$, we will prove (12) for $\operatorname{dim} \tau=k-1$. By Poincaré duality, it suffices to show that the cup product of both sides of (12) with an element of $H^{d(\tau)-i(\sigma), d(\tau)-i(\sigma)}(X \cap V(\tau))$ produces the same result. The cohomology of $X \cap V(\tau)$ decomposes into the toric and residue parts. Using the relations in $H_{\text {toric }}^{*}(X \cap V(\tau))$, coming from the cohomology of the ambient toric variety, we can assume that an element from the toric part is $\varphi_{\tau}^{*} i^{*}[V(\gamma)]$ with $\gamma \in \Sigma(\operatorname{dim} \sigma-\operatorname{dim} \tau)$ such that $\gamma \cap \tau=0$. Then we have

$$
\varphi_{\tau}^{*} i^{*}[V(\gamma)]=\alpha_{\gamma \tau}[X \cap V(\tau+\gamma)]
$$

where $\alpha_{\gamma \tau}=\frac{\operatorname{mult}(\gamma) \operatorname{mult}(\tau)}{\operatorname{mult}(\tau+\gamma)}$ in $H^{*}(X \cap V(\tau))$, because

$$
[V(\tau)] \cdot[V(\gamma)]=\alpha_{\gamma \tau}[X \cap V(\tau+\gamma)]
$$

in the Chow ring $A^{*}\left(\mathbf{P}_{\Sigma}\right)$. Hence,

$$
\varphi_{\tau}^{*} i^{*}[V(\gamma)] \cup \operatorname{Res}_{\tau}(A) \cup \operatorname{Res}_{\tau}(B)=\alpha_{\gamma \tau} \varphi_{\tau+\gamma, \tau !}\left(\varphi_{\tau+\gamma, \tau}^{*} \operatorname{Res}_{\tau}(A) \cup \varphi_{\tau+\gamma, \tau}^{*} \operatorname{Res}_{\tau}(B)\right)
$$

and

$$
\begin{aligned}
\varphi_{\tau}^{*} i^{*}[V(\gamma)] \cup \varphi_{\tau}^{*} i^{*} X^{i(\sigma)-1}=\alpha_{\gamma \tau} \varphi_{\tau+\gamma, \tau !} \varphi_{\tau+\gamma, \tau}^{*} \varphi_{\tau}^{*} i^{*} X^{i(\sigma)-1} & \\
& =\alpha_{\gamma \tau} \varphi_{\tau+\gamma, \tau !} \varphi_{\tau+\gamma}^{*} i^{*} X^{i(\sigma)-1}
\end{aligned}
$$

We can assume that $\tau+\gamma$ forms a cone in $\Sigma$, and that $\gamma$ lies in $\sigma$, because, otherwise, $\varphi_{\tau+\gamma, \tau}^{*} \operatorname{Res}_{\tau}(A)=0$ by the same argument as in [M1, page 102], and $\varphi_{\tau+\gamma, \tau !} \varphi_{\tau+\gamma}^{*} i^{*} X^{i(\sigma)-1}=0$ by Lemma 1.5. In this case,

$$
\begin{aligned}
\varphi_{\tau+\gamma, \tau}^{*} \operatorname{Res}_{\tau}(A) \cup \varphi_{\tau+\gamma, \tau}^{*} \operatorname{Res}_{\tau}(B)=\operatorname{Res}_{\tau+\gamma}(A) \cup & \operatorname{Res}_{\tau+\gamma}(B) \\
& =c_{p}^{\sigma} \operatorname{Res}^{\sigma}(A B) \varphi_{\tau+\gamma}^{*} i^{*} X^{i(\sigma)-1}
\end{aligned}
$$

by the induction assumption. To finish the induction proof of (12), we also need to consider the elements $h \in H_{\text {res }}^{*}(X \cap V(\tau))$, the cup product of which vanishes with the right hand side of (12), by equation (10). The same should hold for the 
left hand side of (12). By part (i) of this proposition, there are two possibilities: $h=\varphi_{\tau, \tau^{\prime} !} h^{\prime}$ with $h^{\prime} \in H_{\text {res }}^{*}\left(X \cap V\left(\tau^{\prime}\right)\right)$ or $h=\operatorname{Res}_{\tau}(C)$. In the first case,

$$
\varphi_{\tau, \tau^{\prime} !} h^{\prime} \cup \operatorname{Res}_{\tau}(A) \cup \operatorname{Res}_{\tau}(B)=\varphi_{\tau, \tau^{\prime} !}\left(h^{\prime} \cup \varphi_{\tau, \tau^{\prime}}^{*} \operatorname{Res}_{\tau}(A) \cup \varphi_{\tau, \tau^{\prime}}^{*} \operatorname{Res}_{\tau}(B)\right) .
$$

If $\tilde{\pi}\left(\tau^{\prime}\right) \not \subset \sigma$, then $\varphi_{\tau, \tau^{\prime}}^{*} \operatorname{Res}_{\tau}(A)=0$ as above. If $\tilde{\pi}\left(\tau^{\prime}\right) \subset \sigma$, then we can apply the induction assumption to deduce that

$$
\varphi_{\tau, \tau^{\prime}}^{*} \operatorname{Res}_{\tau}(A) \cup \varphi_{\tau, \tau^{\prime}}^{*} \operatorname{Res}_{\tau}(B)=\operatorname{Res}_{\tau^{\prime}}(A) \cup \operatorname{Res}_{\tau^{\prime}}(B)
$$

belongs to $H_{\text {toric }}^{*}\left(X \cap V\left(\tau^{\prime}\right)\right)$, the cup product of which with $h^{\prime}$ is zero. In the second case, we have

$$
\operatorname{Res}_{\tau}(A) \cup \operatorname{Res}_{\tau}(B) \cup \operatorname{Res}_{\tau}(C) \in H^{d(\tau)-1, d(\tau)-1}(X \cap V(\tau))
$$

where $d(\tau)=d-\operatorname{dim} \tau$. Up to a constant multiple, the cocycle that represents this cup product is

$$
\left\{A B C \frac{K_{i_{p}} \cdots K_{i_{0}} \Omega}{f_{i_{0}} \cdots f_{i_{p}}} \wedge \frac{K_{i_{i(\sigma)-1}} \cdots K_{i_{p}} \Omega}{f_{i_{p}} \cdots f_{i_{i(\sigma)-1}}} \wedge \frac{K_{i_{d(\tau)-1} \cdots K_{i_{i(\sigma)-1}} \Omega}}{f_{i_{i(\sigma)-1}} \cdots f_{i_{d(\tau)-1}}}\right\}_{i_{0} \cdots i_{d(\tau)-1}}
$$

in $C^{d(\tau)-1}\left(\left.\mathcal{U}\right|_{X \cap V(\tau)}, \Omega_{X \cap V(\tau)}^{d(\tau)-1}\right)$. It follows from [G, Proposition 5.3] that there are $i-\operatorname{dim} \sigma$ open sets from the open cover $\left.\mathcal{U}\right|_{X \cap V(\tau)}$ which cover $X \cap V(\tau)$. Passing to this refinement, we conclude that the above cup product vanishes, since $d-\operatorname{dim} \tau>$ $i-\operatorname{dim} \sigma$. Thus, the equation (12) is shown. The proof of $\operatorname{Res}_{\tau}(A) \cup \operatorname{Res}_{\tau}(B)=0$, for $p+q \neq i-\operatorname{dim} \sigma-1$, follows the same steps as above.

When $\operatorname{dim} \sigma=i-1$, we will use a different strategy. The cup product $\operatorname{Res}_{\tau}(A) \cup$ $\operatorname{Res}_{\tau}(B)$ is represented by the Čech cocycle

$$
\left\{\frac{A B\left\langle m_{1}^{\sigma}, e_{i_{0}}\right\rangle^{2}\left(\prod_{\tilde{\pi}\left(\rho_{k}\right) \not \subset \sigma} x_{k}\right)^{2}}{\left(x_{i_{0}} f_{i_{0}}\right)^{2}}\right\}_{i_{0}} .
$$

Write

$$
A B \prod_{\tilde{\pi}\left(\rho_{k}\right) \not \subset \sigma} x_{k}=P+\operatorname{Res}^{\sigma}(A B) J_{\sigma}
$$

where $P$ is the polynomial determined by (8) with $C=A B$. Using the definition of the projection

$$
p_{\sigma}:\left(S /\left\langle x_{k}: \tilde{\pi}\left(\rho_{k}\right) \subset \sigma\right\rangle\right)_{2 \beta-2 \beta_{0}+2 \beta_{1}^{\sigma}} \rightarrow R_{1}^{\sigma}(f)_{\beta-\beta_{0}+\beta_{1}^{\sigma}}
$$

before this proposition, one can see that the cocycle

$$
\left\{\frac{P\left\langle m_{1}^{\sigma}, e_{i_{0}}\right\rangle^{2} \prod_{\tilde{\pi}\left(\rho_{k}\right) \not \subset \sigma} x_{k}}{\left(x_{i_{0}} f_{i_{0}}\right)^{2}}\right\}_{i_{0}}
$$

is equal to

$$
\left\{\frac{p_{\sigma}(A B)\left\langle m_{1}^{\sigma}, e_{i_{0}}\right\rangle \prod_{\tilde{\pi}\left(\rho_{k}\right) \not \subset \sigma} x_{k}}{x_{i_{0}} f_{i_{0}}}\right\}_{i_{0}}
$$

because the difference is by multiples of $f$ and $x_{k}$, such that $\tilde{\pi}\left(\operatorname{int} \rho_{k}\right) \subset \operatorname{int} \sigma$ and $\rho_{k} \subset \tau$, which vanish on $X \cap V(\tau)$. By Proposition 2.3, this cocycle represents $\operatorname{Res}_{\tau}\left(p_{\sigma}(A B)\right)$. Therefore, $\operatorname{Res}_{\tau}(A) \cup \operatorname{Res}_{\tau}(B)-\operatorname{Res}_{\tau}\left(p_{\sigma}(A B)\right)$ is represented by the cocycle

$$
\left\{\frac{\operatorname{Res}^{\sigma}(A B) J_{\sigma}\left\langle m_{1}^{\sigma}, e_{i_{0}}\right\rangle^{2} \prod_{\tilde{\pi}\left(\rho_{k}\right) \not \subset \sigma} x_{k}}{\left(x_{i_{0}} f_{i_{0}}\right)^{2}}\right\}_{i_{0}}
$$


As above, we can change $J_{\sigma}$ by multiples of $f$ and $x_{k}$, such that $\tilde{\pi}\left(\operatorname{int} \rho_{k}\right) \subset \operatorname{int} \sigma$ and $\rho_{k} \subset \tau$, because they vanish on $X \cap V(\tau)$. Also, we can assume that the index $i_{0}$ takes values in $\left\{s_{1}, s_{2}\right\}$ for $c_{s_{1}}^{\beta, \sigma} s_{2} \neq 0$, since $x_{s_{1}} f_{s_{1}}$ and $x_{s_{2}} f_{s_{2}}$ do not vanish simultaneously on $V(\tau)$, by [M1, Lemma 4.10]. Using an Euler formula, similar to the one in the proof of Proposition 4.7 in M1, we get that

$$
c_{s_{1} s_{2}}^{\beta, \sigma} f-\left\langle m_{1}^{\sigma}, e_{s_{2}}\right\rangle x_{s_{1}} f_{s_{1}}+\left\langle m_{1}^{\sigma}, e_{s_{1}}\right\rangle x_{s_{2}} f_{s_{2}}
$$

with $c_{s_{1} s_{2}}^{\beta, \sigma} \neq 0$ is a multiple of $x_{k}$, such that $\tilde{\pi}\left(\operatorname{int} \rho_{k}\right) \subset \operatorname{int} \sigma$ and $\rho_{k} \subset \tau$. Hence, modulo these multiples and multiples of $f$,

$$
\begin{aligned}
& J_{\sigma}=\frac{\operatorname{det}\left(\begin{array}{cc}
\left(x_{s_{1}} \partial_{s_{1}}\right)^{2} f & \left(x_{s_{1}} \partial_{s_{1}}\right)\left(x_{s_{2}} \partial_{s_{2}}\right) f \\
\left(x_{s_{2}} \partial_{s_{2}}\right)\left(x_{s_{1}} \partial_{s_{1}}\right) f & \left(x_{s_{2}} \partial_{s_{2}}\right)^{2} f
\end{array}\right)}{\left(c_{s_{1} s_{2}}^{\beta, \sigma}\right)^{2} \prod_{\tilde{\pi}\left(\rho_{k}\right) \not \subset \sigma} x_{k}} \\
& \equiv \frac{\operatorname{det}\left(\begin{array}{cc}
x_{s_{1}} f_{s_{1}} & x_{s_{2}} f_{s_{2}} \\
x_{s_{1}} x_{s_{2}} f_{s_{1} s_{2}} & x_{s_{2}} f_{s_{2}}+x_{s_{2}}^{2} f_{s_{2} s_{2}}
\end{array}\right)}{\left\langle m_{1}^{\sigma}, e_{s_{2}}\right\rangle c_{s_{1} s_{2}}^{\beta, \sigma} \prod_{\tilde{\pi}\left(\rho_{k}\right) \not \subset \sigma} x_{k}} \equiv \frac{\operatorname{det}\left(\begin{array}{cc}
f & x_{s_{2}} f_{s_{2}} \\
x_{s_{2}} f_{s_{2}} & x_{s_{2}} f_{s_{2}}+x_{s_{2}}^{2} f_{s_{2} s_{2}}
\end{array}\right)}{\left\langle m_{1}^{\sigma}, e_{s_{2}}\right\rangle^{2} \prod_{\tilde{\pi}\left(\rho_{k}\right) \not \subset \sigma} x_{k}} \\
& \equiv-\frac{\left(x_{s_{2}} f_{s_{2}}\right)^{2}}{\left\langle m_{1}^{\sigma}, e_{s_{2}}\right\rangle^{2} \prod_{\tilde{\pi}\left(\rho_{k}\right) \not \subset \sigma} x_{k}} \equiv-\frac{\left(x_{s_{1}} f_{s_{1}}\right)^{2}}{\left\langle m_{1}^{\sigma}, e_{s_{1}}\right\rangle^{2} \prod_{\tilde{\pi}\left(\rho_{k}\right) \not \subset \sigma} x_{k}} .
\end{aligned}
$$

Therefore, the cocycle (13) coincides with $\left\{-\operatorname{Res}^{\sigma}(A B)\right\}_{i_{0}}$, which comes from the restriction of the global constant function $-\operatorname{Res}^{\sigma}(A B) \in H^{0}\left(V(\tau), \mathcal{O}_{V(\tau)}\right) \cong \mathbb{C}$. We have a commutative diagram:

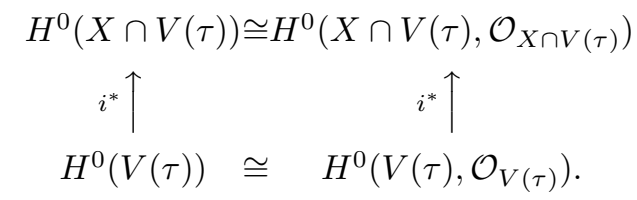

The isomorphism on the bottom of this diagram sends the fundamental class $[V(\tau)]$ to 1 , whence the isomorphism on the top sends $-\operatorname{Res}^{\sigma}(A B) i^{*}[V(\tau)]$ to the element represented by the cocycle (13). This finishes the proof of the proposition.

After all the intermediate technical steps we can describe the generators which span the residue part of the cohomology of semiample nondegenerate hypersurfaces.

Proposition 2.8. Let $X \subset \mathbf{P}_{\Sigma}$ be an i-semiample nondegenerate hypersurface defined by $f \in S_{\beta}$. Then

$$
H_{\mathrm{res}}^{p, q}(X) \cong \sum_{\substack{\sigma \in \Sigma_{X} \\ \gamma \in \Sigma\left(\left(\operatorname{dim}_{\tilde{\pi}(\sin \gamma) \operatorname{c+q}(1-i) / 2)} \operatorname{int} \sigma\right.\right.}} \varphi_{\gamma !} \operatorname{Res}_{\gamma} R_{1}^{\sigma}(f)_{(q+1-\operatorname{dim} \gamma) \beta-\beta_{0}+\beta_{1}^{\sigma}}
$$

Proof. We can actually prove by induction on the codimension of $\tau \in \Sigma$ a more general statement:

$$
H_{\mathrm{res}}^{p, q}(X \cap V(\tau)) \cong \sum_{\substack{\tilde{\pi}(\tau) \subset \sigma \in \Sigma_{X} \\ \tau \subset \gamma \in \Sigma((p+q+1-i(\sigma)) / 2+\operatorname{dim} \tau) \\ \tilde{\pi}(\operatorname{int} \gamma) \subset \operatorname{int} \sigma}} \varphi_{\tau, \gamma !} \operatorname{Res}_{\gamma} R_{1}^{\sigma}(f)_{(q+1-\operatorname{dim} \gamma+\operatorname{dim} \tau) \beta-\beta_{0}+\beta_{1}^{\sigma}}
$$

This is certainly true for the maximally dimensional $\tau \in \Sigma(d)$, because $X \cap V(\tau)$ is empty by Proposition 1.6 and Remark 2.2 in M2 and there is no $\gamma$ satisfying the conditions on the right side of the formula. Assume that the formula holds for 
$\tau \in \Sigma(k-1)$. Then Proposition 2.7 and the induction assumption produce the result for $\tau \in \Sigma(k)$.

The next result describes the product structure on the residue part of the cohomology of semiample nondegenerate hypersurfaces.

Proposition 2.9. Let $X \subset \mathbf{P}_{\Sigma}$ be an i-semiample nondegenerate hypersurface defined by $f \in S_{\beta}$, then:

(i) for $\sigma \in \Sigma_{X}$ with $\operatorname{dim} \sigma<i-1, \gamma_{1}, \gamma_{2} \in \Sigma$, such that $\tilde{\pi}\left(\operatorname{int} \gamma_{k}\right) \subset \operatorname{int} \sigma$ with $k=1,2$, and $A \in R_{1}^{\sigma}(f)_{(p+1) \beta-\beta_{0}+\beta_{1}^{\sigma}}, B \in R_{1}^{\sigma}(f)_{* \beta-\beta_{0}+\beta_{1}^{\sigma}}$,

$$
\frac{\varphi_{\gamma_{1} !} \operatorname{Res}_{\gamma_{1}}(A)}{\operatorname{mult}\left(\gamma_{1}\right)} \cup \frac{\varphi_{\gamma_{2} !} \operatorname{Res}_{\gamma_{2}}(B)}{\operatorname{mult}\left(\gamma_{2}\right)}=c_{p}^{\sigma} \operatorname{Res}^{\sigma}(A B) i^{*} X^{i(\sigma)-1} \prod_{\rho_{k} \subset \gamma_{1}} i^{*} D_{k} \prod_{\rho_{k} \subset \gamma_{2}} i^{*} D_{k},
$$

where $i(\sigma)=i-\operatorname{dim} \sigma$ and

$$
c_{p}^{\sigma}=-(-2 \pi \sqrt{-1})^{i(\sigma)-1} \frac{(-1)^{(i(\sigma)-1)(i(\sigma)+2 p+2)}}{p !(i(\sigma)-p-1) !},
$$

(ii) for $\operatorname{dim} \sigma=i-1, A, B$ and $\gamma_{1}, \gamma_{2}$, as in part (i),

$$
\begin{aligned}
\frac{\varphi_{\gamma_{1} !} \operatorname{Res}_{\gamma_{1}}(A)}{\operatorname{mult}\left(\gamma_{1}\right)} \cup \frac{\varphi_{\gamma_{2} !} \operatorname{Res}_{\gamma_{2}}(B)}{\operatorname{mult}\left(\gamma_{2}\right)}=- & \operatorname{Res}^{\sigma}(A B) i^{*}\left[\mathbf{P}_{\Sigma}\right] \prod_{\rho_{k} \subset \gamma_{1}} i^{*} D_{k} \prod_{\rho_{k} \subset \gamma_{2}} i^{*} D_{k} \\
& +\prod_{\rho_{k} \subset \gamma_{1} \cap \gamma_{2}} i^{*} D_{k} \frac{\varphi_{\gamma_{1}+\gamma_{2} !} \operatorname{Res}_{\gamma_{1}+\gamma_{2}}\left(p_{\sigma}(A B)\right)}{\operatorname{mult}\left(\gamma_{1}+\gamma_{2}\right)},
\end{aligned}
$$

(iii) for $\gamma_{1}, \gamma_{2} \in \Sigma$ satisfying neither part (i) nor (ii),

$$
\varphi_{\gamma_{1} !} \operatorname{Res}_{\gamma_{1}}(A) \cup \varphi_{\gamma_{2} !} \operatorname{Res}_{\gamma_{2}}(B)=0 .
$$

Proof. Applying the Gysin projection formula, we get

$$
\begin{aligned}
\varphi_{\gamma_{1} !} & \operatorname{Res}_{\gamma_{1}}(A) \cup \varphi_{\gamma_{2} !} \operatorname{Res}_{\gamma_{2}}(B) \\
& =\varphi_{\gamma_{1} \cap \gamma_{2} !} \varphi_{\gamma_{1} \cap \gamma_{2}, \gamma_{1} !} \operatorname{Res}_{\gamma_{1}}(A) \cup \varphi_{\gamma_{1} \cap \gamma_{2} !} \varphi_{\gamma_{1} \cap \gamma_{2}, \gamma_{2} !} \operatorname{Res}_{\gamma_{2}}(B) \\
& =\varphi_{\gamma_{1} \cap \gamma_{2} !}\left(\varphi_{\gamma_{1} \cap \gamma_{2}}^{*} \varphi_{\gamma_{1} \cap \gamma_{2} !} \varphi_{\gamma_{1} \cap \gamma_{2}, \gamma_{1} !} \operatorname{Res}_{\gamma_{1}}(A) \cup \varphi_{\gamma_{1} \cap \gamma_{2}, \gamma_{2} !} \operatorname{Res}_{\gamma_{2}}(B)\right) \\
& =\varphi_{\gamma_{1} \cap \gamma_{2} !} \varphi_{\gamma_{1} \cap \gamma_{2}}^{*} \varphi_{\gamma_{1} \cap \gamma_{2} !}\left(\varphi_{\gamma_{1} \cap \gamma_{2}, \gamma_{1} !} \operatorname{Res}_{\gamma_{1}}(A) \cup \varphi_{\gamma_{1} \cap \gamma_{2}, \gamma_{2} !} \operatorname{Res}_{\gamma_{2}}(B)\right) \\
& =\varphi_{\gamma_{1} \cap \gamma_{2} !} \varphi_{\gamma_{1} \cap \gamma_{2}}^{*} \varphi_{\gamma_{1} \cap \gamma_{2} !} \varphi_{\gamma_{1} \cap \gamma_{2}, \gamma_{2} !}\left(\varphi_{\gamma_{1} \cap \gamma_{2}, \gamma_{2}}^{*} \varphi_{\gamma_{1} \cap \gamma_{2}, \gamma_{1} !} \operatorname{Res}_{\gamma_{1}}(A) \cup \operatorname{Res}_{\gamma_{2}}(B)\right) \\
& =\varphi_{\gamma_{1} \cap \gamma_{2} !} \varphi_{\gamma_{1} \cap \gamma_{2}}^{*} \varphi_{\gamma_{2} !}\left(\alpha_{\gamma_{1} \gamma_{2}} \varphi_{\gamma_{2}, \gamma_{1}+\gamma_{2} !} \varphi_{\gamma_{1}, \gamma_{1}+\gamma_{2}}^{*} \operatorname{Res}_{\gamma_{1}}(A) \cup \operatorname{Res}_{\gamma_{2}}(B)\right) \\
& =\alpha_{\gamma_{1} \gamma_{2}} \varphi_{\gamma_{1} \cap \gamma_{2} !} \varphi_{\gamma_{1} \cap \gamma_{2}}^{*} \varphi_{\gamma_{2} !} \varphi_{\gamma_{2}, \gamma_{1}+\gamma_{2} !}\left(\varphi_{\gamma_{1}, \gamma_{1}+\gamma_{2}}^{*} \operatorname{Res}_{\gamma_{1}}(A) \cup \varphi_{\gamma_{2}, \gamma_{1}+\gamma_{2}}^{*} \operatorname{Res}_{\gamma_{2}}(B)\right) \\
& =\alpha_{\gamma_{1} \gamma_{2}} \varphi_{\gamma_{1} \cap \gamma_{2} !} \varphi_{\gamma_{1} \cap \gamma_{2}}^{*} \varphi_{\gamma_{1}+\gamma_{2} !}\left(\varphi_{\gamma_{1}, \gamma_{1}+\gamma_{2}}^{*} \operatorname{Res}_{\gamma_{1}}(A) \cup \varphi_{\gamma_{2}, \gamma_{1}+\gamma_{2}}^{*} \operatorname{Res}_{\gamma_{2}}(B)\right) \\
& =\alpha_{\gamma_{1} \gamma_{2}} i^{*}\left[V\left(\gamma_{1} \cap \gamma_{2}\right)\right] \varphi_{\gamma_{1}+\gamma_{2} !}\left(\varphi_{\gamma_{1}, \gamma_{1}+\gamma_{2}}^{*} \operatorname{Res}_{\gamma_{1}}(A) \cup \varphi_{\gamma_{2}, \gamma_{1}+\gamma_{2}} \operatorname{Res}_{\gamma_{2}}(B)\right),
\end{aligned}
$$

where we also used the commutative diagram (see [M1, Lemma 5.4]):

$$
\begin{array}{ccc}
H^{*}\left(X \cap V\left(\gamma_{1}\right)\right) & \stackrel{\varphi_{\gamma_{1} \cap \gamma_{2}, \gamma_{1} !}}{\longrightarrow} & H^{*}\left(X \cap V\left(\gamma_{1} \cap \gamma_{2}\right)\right) \\
\varphi_{\gamma_{1}, \gamma_{1}+\gamma_{2}}^{*} & & \varphi_{\gamma_{1} \cap \gamma_{2}}^{*} \downarrow \\
H^{*}\left(X \cap V\left(\gamma_{1}+\gamma_{2}\right)\right) \stackrel{\alpha_{\gamma_{1} \gamma_{2} \varphi_{\gamma_{2}, \gamma_{1}+\gamma_{2} !}}^{\longrightarrow}}{ } & H^{*}\left(X \cap V\left(\gamma_{2}\right)\right),
\end{array}
$$

with $\alpha_{\gamma_{1} \gamma_{2}}$ satisfying the equality $\left[X \cap V\left(\gamma_{1}\right)\right] \cup\left[X \cap V\left(\gamma_{2}\right)\right]=\alpha_{\gamma_{1} \gamma_{2}}\left[X \cap V\left(\gamma_{1}+\gamma_{2}\right)\right]$ in $H^{*}\left(X \cap V\left(\gamma_{1} \cap \gamma_{2}\right)\right)$.

Note that the cup product calculated above is zero, if $\gamma_{1}$ and $\gamma_{2}$ do not span a cone in $\Sigma$. It also vanishes when $\gamma_{1}$ and $\gamma_{2}$ have different smallest cones $\sigma_{1}$ and 
$\sigma_{2}$ in $\Sigma_{X}$ containing them. Indeed, we can assume that $\sigma_{1}$ is not contained in $\sigma_{2}$. Then $\varphi_{\gamma_{1}, \gamma_{1}+\gamma_{2}}^{*} \operatorname{Res}_{\gamma_{1}}(A)$ vanishes by the same argument as in [M1, p. 102].

To determine the constant $\alpha_{\gamma_{1} \gamma_{2}}$ first notice that $[X \cap V(\gamma)]=i^{*}[V(\gamma)]$, for $\gamma \in \Sigma$, by the arguments in the proof of Lemma 5.7 in [M1]. On the other hand, we can write $\gamma_{k}=\gamma_{k}^{\prime}+\gamma_{1} \cap \gamma_{2}$, for $k=1,2$, so that $\gamma^{\prime} \in \Sigma$ and $\operatorname{dim} \gamma_{k}=$ $\operatorname{dim} \gamma_{k}^{\prime}+\operatorname{dim}\left(\gamma_{1} \cap \gamma_{2}\right)$. In this case,

$$
[V(\gamma)]=\frac{\operatorname{mult}(\gamma)}{\operatorname{mult}\left(\gamma^{\prime}\right) \operatorname{mult}\left(\gamma_{1} \cap \gamma_{2}\right)} \varphi_{\gamma_{1} \cap \gamma_{2}}^{*}\left[V\left(\gamma^{\prime}\right)\right]
$$

in $H^{*}\left(V\left(\gamma_{1} \cap \gamma_{2}\right)\right)$, where $\gamma$ is $\gamma_{1}, \gamma_{2}$ or $\gamma_{1}+\gamma_{2}$ and $\gamma^{\prime}$ is $\gamma_{1}^{\prime}, \gamma_{2}^{\prime}$ or $\gamma_{1}^{\prime}+\gamma_{2}^{\prime}$, respectively. This is because

$$
V(\gamma)=\frac{\operatorname{mult}(\gamma)}{\operatorname{mult}\left(\gamma^{\prime}\right) \operatorname{mult}\left(\gamma_{1} \cap \gamma_{2}\right)} V\left(\gamma_{1} \cap \gamma_{2}\right) \cdot V\left(\gamma^{\prime}\right)
$$

in the Chow ring $A^{*}\left(\mathbf{P}_{\Sigma}\right)$ for the corresponding $\gamma$ and $\gamma^{\prime}$. Since

$$
V\left(\gamma_{1}^{\prime}\right) \cdot V\left(\gamma_{2}^{\prime}\right)=\frac{\operatorname{mult}\left(\gamma_{1}^{\prime}\right) \operatorname{mult}\left(\gamma_{2}^{\prime}\right)}{\operatorname{mult}\left(\gamma_{1}^{\prime}+\gamma_{2}^{\prime}\right)} V\left(\gamma_{1}^{\prime}+\gamma_{2}^{\prime}\right)
$$

it is not difficult to calculate

$$
\alpha_{\gamma_{1} \gamma_{2}}=\frac{\operatorname{mult}\left(\gamma_{1}\right) \operatorname{mult}\left(\gamma_{2}\right)}{\operatorname{mult}\left(\gamma_{1} \cap \gamma_{2}\right) \operatorname{mult}\left(\gamma_{1}+\gamma_{2}\right)} .
$$

Hence,

$$
\begin{aligned}
& \varphi_{\gamma_{1} !} \operatorname{Res}_{\gamma_{1}}(A) \cup \varphi_{\gamma_{2} !} \operatorname{Res}_{\gamma_{2}}(B) \\
& \quad=\frac{\operatorname{mult}\left(\gamma_{1}\right) \operatorname{mult}\left(\gamma_{2}\right)}{\operatorname{mult}\left(\gamma_{1}+\gamma_{2}\right)} \prod_{\rho_{k} \subset \gamma_{1} \cap \gamma_{2}} i^{*} D_{k} \varphi_{\gamma_{1}+\gamma_{2} !}\left(\operatorname{Res}_{\gamma_{1}+\gamma_{2}}(A) \cup \operatorname{Res}_{\gamma_{1}+\gamma_{2}}(B)\right)
\end{aligned}
$$

Applying Proposition 2.8, we get the result.

To give a very explicit description of the cohomology of semiample hypersurfaces we introduce the following rings.

Definition 2.10. Given a semiample class $[D] \in A_{d-1}\left(\mathbf{P}_{\Sigma}\right)$ and $\sigma \in \Sigma_{D}$, let

$$
U^{\sigma}(D)=\left\langle\prod_{\rho_{k} \subset \gamma \in \Sigma} D_{k}: \tilde{\pi}(\operatorname{int} \gamma) \subset \operatorname{int} \sigma\right\rangle
$$

be the ideal in $\mathbb{C}\left[D_{1}, \ldots, D_{n}\right]$. Define the bigraded ring

$A_{1}^{\sigma}(D)_{*, *}=U^{\sigma}(D) /\left\{u \in U^{\sigma}(D): u v X^{i(\sigma)} \in(P(\Sigma)+S R(\Sigma))\right.$ for all $\left.v \in U^{\sigma}(D)\right\}$, where $D_{k}$ has the degree $(1,1)$.

Here is our main result.

Theorem 2.11. Let $X \subset \mathbf{P}_{\Sigma}$ be an i-semiample nondegenerate hypersurface defined by $f \in S_{\beta}$. Then there is a ring isomorphism

$$
\bigoplus_{p, q} H^{p, q}(X) \cong \bigoplus_{p, q} A_{1}(X)_{p, q} \oplus\left(\bigoplus_{\sigma \in \Sigma_{X}} A_{1}^{\sigma}(X)_{s, s} \otimes R_{1}^{\sigma}(f)_{(q-s+1) \beta-\beta_{0}+\beta_{1}^{\sigma}}\right),
$$

where $s=(p+q-i-\operatorname{dim} \sigma+1) / 2$. The product structure on the right side is given by:

(a) $a \cdot b=a b$ for $a, b \in A_{1}(X)$,

(b) $a \cdot(u \otimes g)=(a u) \otimes g$ for $a \in A_{1}(X), u \in A_{1}^{\sigma}(X), g \in R_{1}^{\sigma}(f)_{* \beta-\beta_{0}+\beta_{1}^{\sigma}}$, 
(c) $(u \otimes g) \cdot(v \otimes h)=c_{r}^{\sigma} \operatorname{Res}^{\sigma}(g h) X^{i(\sigma)-1} u v$ for $\operatorname{dim} \sigma<i-1, u, v \in A_{1}^{\sigma}(X)$, $g \in R_{1}^{\sigma}(f)_{(r+1) \beta-\beta_{0}+\beta_{1}^{\sigma}}, h \in R_{1}^{\sigma}(f)_{t \beta-\beta_{0}+\beta_{1}^{\sigma}}\left(\operatorname{Res}^{\sigma}(g h)\right.$ vanishes unless $r+t=i(\sigma)$, $i(\sigma)=i-\operatorname{dim} \sigma)$,

(d) $(u \otimes g) \cdot(v \otimes h)=-\operatorname{Res}^{\sigma}(g h) u v+(u v) \otimes p_{\sigma}(g h)$ for $\operatorname{dim} \sigma=i-1, u, v, g, h$, as in part (b),

(e) $(u \otimes g) \cdot(v \otimes h)=0$ if $u \otimes g$ and $v \otimes h$ belong to the factors in the decomposition, corresponding to distinct cones in $\Sigma_{X}$.

Proof. The first factor $A_{1}(X)_{p, q}$ in the decomposition of $H^{p, q}(X)$ is the toric part of the cohomology. We will prove that the rest is isomorphic to the residue part.

Let $u \in U^{\sigma}(X)$ and $g \in R_{1}^{\sigma}(f)_{* \beta-\beta_{0}+\beta_{1}^{\sigma}}$. By the definition of $U^{\sigma}(X)$, we can assume that $u=b \cdot \prod_{\rho_{k} \subset \gamma} i^{*} D_{k}$ for some $b \in U^{\sigma}(X)$ and $\gamma \in \Sigma$ such that $\tilde{\pi}\left(\operatorname{int} \gamma_{k}\right) \subset$ $\operatorname{int} \sigma$. The map assigning $b \varphi_{\gamma !} \operatorname{Res}_{\gamma}(g)$ to $u \otimes g$ is well defined, because

$$
\frac{\varphi_{\gamma !} \operatorname{Res}_{\gamma}(A)}{\operatorname{mult}(\gamma)}=\prod_{\rho_{k} \subset \gamma^{\prime} \backslash \gamma} i^{*} D_{k} \varphi_{\gamma^{\prime} !} \operatorname{Res}_{\gamma^{\prime}}(A)
$$

for any $\gamma^{\prime} \subset \gamma$ such that $\tilde{\pi}\left(\operatorname{int} \gamma^{\prime}\right) \subset \operatorname{int} \sigma$ and $\tilde{\pi}(\operatorname{int} \gamma) \subset \operatorname{int} \sigma$. To show this use the Gysin projection formula as in the proof of Proposition 2.9. We omit these details. Hence, by Proposition 2.8, we have a surjective map

$$
\bigoplus_{\sigma \in \Sigma_{X}} U^{\sigma}(X)_{s, s} \otimes R_{1}^{\sigma}(f)_{(q-s+1) \beta-\beta_{0}+\beta_{1}^{\sigma}} \stackrel{\psi}{\rightarrow} H_{\mathrm{res}}^{p, q}(X),
$$

where $s=(p+q-i-\operatorname{dim} \sigma+1) / 2$. To find the kernel of this map we use the Poincaré duality. This duality and Proposition 2.9(iii) show that the kernel cannot intersect the sum of two or more factors on the left side of (14). Therefore, we can assume that a given element from the kernel belongs to one of the factors $U^{\sigma}(X)_{s, s} \otimes R_{1}^{\sigma}(f)_{(q-s+1) \beta-\beta_{0}+\beta_{1}^{\sigma}}$ for some $\sigma \in \Sigma_{X}$. Let $\sum_{k=1}^{l} u_{k} \otimes g_{k}$ be this element, where $g_{1}, \ldots, g_{l}$ is a basis of $R_{1}^{\sigma}(f)_{(q-s+1) \beta-\beta_{0}+\beta_{1}^{\sigma}}$, and $u_{k} \in U^{\sigma}(X)_{s, s}$. The composition

$R_{1}^{\sigma}(f)_{(q-s+1) \beta-\beta_{0}+\beta_{1}^{\sigma}} \otimes R_{1}^{\sigma}(f)_{(i(\sigma)-q+s) \beta-\beta_{0}+\beta_{1}^{\sigma}} \rightarrow R_{1}^{\sigma}(f)_{(i(\sigma)+1) \beta-2 \beta_{0}+2 \beta_{1}^{\sigma}} \stackrel{\operatorname{Res}^{\sigma}}{\longrightarrow} \mathbb{C}$,

where the first arrow is a multiplication, is a nondegenerate pairing because of Proposition 2.2(ii), [M1, Theorem 4.4] and the definition of Res ${ }^{\sigma}$. Hence, there is a basis $g_{1}^{\prime}, \ldots, g_{l}^{\prime}$ of $R_{1}^{\sigma}(f)_{(i(\sigma)-q+s) \beta-\beta_{0}+\beta_{1}^{\sigma}}$, orthogonal to $g_{1}, \ldots, g_{l}$ with respect to this pairing. The fact that $\psi\left(\sum_{k=1}^{l} u_{k} \otimes g_{k}\right)=0$ is equivalent to $\int_{X} \psi\left(\sum_{k=1}^{l} u_{k} \otimes\right.$ $\left.g_{k}\right) \cup t=0$ for all $t \in H^{*}(X)$. This is true for $t \in H_{\text {toric }}^{*}(X)$, and, by Proposition 2.9(iii), we can consider only $t=\psi\left(v \otimes g_{k}^{\prime}\right)$ with $v \in U^{\sigma}(X)_{d-i(\sigma)-s, d-i(\sigma)-s}$ and $k=1, \ldots, l$. Applying the parts (i) and (ii) of Proposition 2.9, we get

$$
\int_{X} \psi\left(\sum_{k=1}^{l} u_{k} \otimes g_{k}\right) \cup \psi\left(v \otimes g_{k}^{\prime}\right)=\int_{\mathbf{P}_{\Sigma}} c_{q-s}^{\sigma} \operatorname{Res}^{\sigma}\left(g_{k} g_{k}^{\prime}\right) X^{i(\sigma)} u_{k} v .
$$

Hence, $\psi\left(\sum_{k=1}^{l} u_{k} \otimes g_{k}\right)=0$ if and only if $X^{i(\sigma)} u_{k} v \in(P(\Sigma)+S R(\Sigma))$ for all $k$ and $v \in U^{\sigma}(X)_{d-i(\sigma)-s, d-i(\sigma)-s}$. In the last condition we can take all $v \in U^{\sigma}(X)$, because the cohomology $H^{*}\left(\mathbf{P}_{\Sigma}\right) \cong \mathbb{C}\left[D_{1}, \ldots, D_{n}\right] /(P(\Sigma)+S R(\Sigma))$ of the complete simplicial toric variety $\mathbf{P}_{\Sigma}$ satisfies the Poincaré duality. Thus, the decomposition of $H^{*, *}(X)$ is proved, and the product structure on it follows from Proposition 2.9. 


\section{The PiCARD Group of SEMiAmple hypersurfaces.}

In this section we will explicitly describe the Picard group of semiample nondegenerate hypersurfaces in a complete simplicial toric variety. Our approach provides a better description than the one in [R] with complicated restrictions.

First, we have the following property for semiample nondegenerate hypersurfaces.

Lemma 3.1. Let $X$ be an i-semiample nondegenerate hypersurface in a complete simplicial toric variety $\mathbf{P}_{\Sigma}$. Then

$$
H^{k}\left(X, \mathcal{O}_{X}\right)=0 \quad \text { for } k \neq 0, i-1 .
$$

Proof. Since

$$
H^{k}\left(X, \mathcal{O}_{X}\right) \cong H^{0, k}(X) \cong H_{\text {toric }}^{0, k}(X) \oplus H_{\text {res }}^{0, k}(X)
$$

and $H^{0, k}\left(\mathbf{P}_{\Sigma}\right)$ vanishes for $k \neq 0$, the statement is implied by Proposition 2.7.

By this lemma, for an $i$-semiample nondegenerate hypersurface $X$ with $i>3$, we have

$$
H^{1}\left(X, \mathcal{O}_{X}\right)=H^{2}\left(X, \mathcal{O}_{X}\right)=0
$$

Combining this with the standard exponential sequence

$$
0 \rightarrow \mathbb{Z} \rightarrow \mathcal{O}_{X} \stackrel{\exp }{\rightarrow} \mathcal{O}_{X}^{*} \rightarrow 0
$$

is enough to conclude

$$
\operatorname{Pic}(X)_{\mathbb{C}} \cong H^{2}(X, \mathbb{C}) \cong H^{1,1}(X)
$$

Theorem 3.2. Let $X \subset \mathbf{P}_{\Sigma}$ be an $i$-semiample nondegenerate hypersurface defined by $f \in S_{\beta}$, and let $i>3$. Then

$$
\begin{aligned}
\operatorname{Pic}(X)_{\mathbb{C}} & \cong H^{2}(X, \mathbb{C}) \cong H^{1,1}(X) \\
& \cong\left(\bigoplus_{k=1}^{n} \mathbb{C} D_{k}\right) / C \bigoplus\left(\bigoplus_{\sigma \in \Sigma_{X}(i-1)} \bigoplus_{\tilde{\pi}\left(\operatorname{int} \rho_{k}\right) \subset \operatorname{int} \sigma} \mathbb{C} D_{k} \otimes R_{1}^{\sigma}(f)_{\beta-\beta_{0}+\beta_{1}^{\sigma}}\right),
\end{aligned}
$$

where $C:=\operatorname{span}_{\mathbb{C}}\left\{\sum_{k=1}^{n}\left\langle m, e_{k}\right\rangle D_{k}, D_{l}: m \in M, \tilde{\pi}\left(\operatorname{int} \rho_{l}\right) \subset \operatorname{int} \sigma, \sigma \in \Sigma_{X}(i)\right\}$.

Proof. By Theorem 2.11.

$$
H^{1,1}(X) \cong A_{1}(X)_{1,1} \oplus\left(\bigoplus_{\sigma \in \Sigma_{X}(i-1)} A_{1}^{\sigma}(X)_{1,1} \otimes R_{1}^{\sigma}(f)_{\beta-\beta_{0}+\beta_{1}^{\sigma}}\right) .
$$

One may try to use only the definitions of $A_{1}(X)$ and $A_{1}^{\sigma}(X)$ to deduce the result. However, we will use some additional information to alleviate this task. By definition, $A_{1}(X)_{1,1}$ is a quotient of $H^{1,1}\left(\mathbf{P}_{\Sigma}\right) \cong \operatorname{Pic}\left(\mathbf{P}_{\Sigma}\right)_{\mathbb{C}}$, the description of which we know, for instance, from [F]. Also, Proposition 1.6 or Lemma 1.5 imply that $X \cdot D_{l}=0$ in $H^{*}\left(\mathbf{P}_{\Sigma}\right)$ for $\tilde{\pi}\left(\operatorname{int} \rho_{l}\right) \subset \operatorname{int} \sigma, \sigma \in \Sigma_{X}(i)$. This shows that $H^{1,1}(X)$ is at least a quotient space of the given answer. To prove this answer it now suffices to verify that $h^{1,1}(X)$ coincides with

$$
n-d-\sum_{\sigma \in \Sigma_{X}(i)} a_{1}(\sigma)+\sum_{\sigma \in \Sigma_{X}(i-1)} a_{1}(\sigma) \operatorname{dim} R_{1}^{\sigma}(f)_{\beta-\beta_{0}+\beta_{1}^{\sigma}}
$$


where $a_{1}(\sigma)$ is the number of the 1-dimensional cones $\rho_{l}$ such that $\tilde{\pi}\left(\operatorname{int} \rho_{l}\right) \subset \operatorname{int} \sigma$. As in [M1, Section 4], the Gysin spectral sequence gives the exact sequence:

$0 \rightarrow H^{1,1}\left(H^{1}(X \cap \mathbf{T})\right) \rightarrow \bigoplus_{k=1}^{n} H^{0,0}\left(X \cap D_{k}\right) \rightarrow H^{1,1}(X) \rightarrow H^{1,1}\left(H^{2}(X \cap \mathbf{T})\right) \rightarrow 0$.

Since $i>3, H^{1,1}\left(H^{2}(X \cap \mathbf{T})\right)$ vanishes as in the proof of Proposition 2.7(i). On the other side, Theorem 1.7 gives $h^{1,1}\left(H^{1}(X \cap \mathbf{T})\right)=h^{1,1}\left(H^{1}(\mathbf{T})\right)=d$. We also have

$$
H^{0}\left(X \cap D_{k}\right)=H_{\text {toric }}^{0}\left(X \cap D_{k}\right) \oplus H_{\text {res }}^{0}\left(X \cap D_{k}\right) .
$$

The dimension of $H_{\text {toric }}^{0}\left(X \cap D_{k}\right)$ is clearly 1 , when $X \cap D_{k}$ is nonempty. By Proposition 1.6, the last condition is the same as $\tilde{\pi}\left(\operatorname{int} \rho_{k}\right) \subset \operatorname{int} \sigma$ for some $\sigma \in \Sigma_{X}$ of dimension less than $i$. Finally, Proposition 2.7 gives $H_{\mathrm{res}}^{0}\left(X \cap D_{k}\right) \cong R_{1}^{\sigma}(f)_{\beta-\beta_{0}+\beta_{1}^{\sigma}}$, and a simple count verifies the dimension of $H^{1,1}(X)$.

It would actually be convenient for us to use a more geometric description of the Picard group of $X$. The factor $\mathbb{C} D_{k} \otimes R_{1}^{\sigma}(f)_{\beta-\beta_{0}+\beta_{1}^{\sigma}}$ in the above theorem is isomorphic to $H_{\text {res }}^{0}\left(X \cap D_{k}\right)$, while there is an isomorphism

$$
\pi_{\rho_{k}}^{*}: H_{\mathrm{res}}^{0}(Y \cap V(\sigma)) \cong H_{\mathrm{res}}^{0}\left(X \cap D_{k}\right) .
$$

The intersection $Y \cap V(\sigma)$ is a finite set of points $p_{1}, \ldots, p_{\mathrm{vol}\left(\Gamma_{\sigma}\right)}$ inside the 1dimensional toric variety $V(\sigma) \cong \mathbb{P}^{1}$. Hence,

$$
H^{0}(Y \cap V(\sigma)) \cong \bigoplus_{l=1}^{\operatorname{vol}\left(\Gamma_{\sigma}\right)} \mathbb{C} p_{l}
$$

It follows from the definition of the residue part that $H_{\mathrm{res}}^{0}(Y \cap V(\sigma))$ consists of $\sum_{l=1}^{\operatorname{vol}\left(\Gamma_{\sigma}\right)} a_{l} p_{l}$ such that $\sum_{l=1}^{\operatorname{vol}\left(\Gamma_{\sigma}\right)} a_{l}=0$. Correspondingly, we get that $X \cap D_{k}=$ $\bigcup_{l=1}^{\mathrm{vol}\left(\Gamma_{\sigma}\right)} X_{k, l}$ is a disjoint union of $\operatorname{vol}\left(\Gamma_{\sigma}\right)$ irreducible components, and

$$
H_{\mathrm{res}}^{0}\left(X \cap D_{k}\right) \cong\left\{\sum_{l=1}^{\operatorname{vol}\left(\Gamma_{\sigma}\right)} a_{l} X_{k, l}: \sum_{l=1}^{\operatorname{vol}\left(\Gamma_{\sigma}\right)} a_{l}=0\right\} .
$$

Corollary 3.3. Let $X \subset \mathbf{P}_{\Sigma}$ be an i-semiample nondegenerate hypersurface with $i>3$. Then

$$
\operatorname{Pic}(X)_{\mathbb{C}} \cong\left(\bigoplus_{k=1}^{n} \mathbb{C} D_{k}\right) / C \bigoplus\left(\bigoplus_{\substack{\sigma \in \Sigma_{X}(i-1) \\ \tilde{\pi}\left(\operatorname{int} \rho_{k}\right) \subset \operatorname{int} \sigma}}\left\{\sum_{l=1}^{\operatorname{vol}\left(\Gamma_{\sigma}\right)} a_{l} X_{k, l}: \sum_{l=1}^{\operatorname{vol}\left(\Gamma_{\sigma}\right)} a_{l}=0\right\}\right),
$$

where $C$ is as in Theorem 3.9, and $X_{k, l}$ is a connected component of $X \cap D_{k}$.

To connect this description of the Picard group to the one in Theorem 3.2, we will show which elements in the latter correspond to $-X_{k, l}+X_{k, l+1}, l=1, \ldots, \operatorname{vol}\left(\Gamma_{\sigma}\right)-$ 1 , in the former. Why we choose such a basis will be clear when we construct a mirror map in Section 5 .

Let $X$ be determined by $f \in S_{\beta}$ be linearly equivalent to a torus invariant divisor $\sum_{k=1}^{n} b_{k} D_{k}$ with the associated polytope $\Delta_{D}=\left\{m \in M_{\mathbb{R}}:\left\langle m, e_{k}\right\rangle \geq\right.$ $-b_{k}$ for all $\left.k\right\}$. Write $f=\sum_{m \in \Delta \cap M} a_{m} x^{D(m)}$ where $x^{D(m)}=\prod_{k=1}^{n} x_{k}^{b_{k}+\left\langle m, e_{k}\right\rangle}$. The restriction of this polynomial $\left.f\right|_{D_{k}}=\sum_{m \in \Gamma_{\sigma} \cap M} a_{m} x^{D(m)}$ determines $X \cap D_{k}$. Since $m_{1}^{\sigma}$ is a basis element for the lattice $M_{X} \cap \sigma^{\perp}$, the lattice points $m \in \Gamma_{\sigma}$ are 
$m_{0}+s m_{1}^{\sigma}, s=0, \ldots, \operatorname{vol}\left(\Gamma_{\sigma}\right)$, where $m_{0}$ is some of the vertices of $\Gamma_{\sigma}$. Then the polynomial $\left.f\right|_{D_{k}}$ can be factored as

$$
a_{m_{0}+\operatorname{vol}\left(\Gamma_{\sigma}\right) m_{1}^{\sigma}} x^{D\left(m_{0}\right)} \prod_{s=1}^{\operatorname{vol}\left(\Gamma_{\sigma}\right)}\left(\prod_{k=1}^{n} x_{k}^{\left\langle m_{1}^{\sigma}, e_{k}\right\rangle}-\lambda_{s}\right),
$$

where $\prod_{k=1}^{n} x_{k}^{\left\langle m_{1}^{\sigma}, e_{k}\right\rangle}$ is a coordinate on the torus $\mathbf{T}_{\rho_{k}}$, which is equal to $\lambda_{s}$ precisely on the connected component $X_{k, s}$ of $X \cap D_{k}$. This can be seen from the description of the map $\pi$ in Proposition 1.6.

Lemma 3.4. Let $X \subset \mathbf{P}_{\Sigma}$ be an i-semiample nondegenerate hypersurface defined by $f \in S_{\beta}$. Then $-X_{k, l}+X_{k, l+1}, l=1, \ldots, \operatorname{vol}\left(\Gamma_{\sigma}\right)-1$, corresponds to $D_{k} \otimes g_{l}$, where

$$
g_{l}=\frac{\left(\lambda_{l+1}-\lambda_{l}\right) f \prod_{k=1}^{n} x_{k}^{\left\langle m_{1}^{\sigma}, e_{k}\right\rangle}}{\left(\prod_{k=1}^{n} x_{k}^{\left\langle m_{1}^{\sigma}, e_{k}\right\rangle}-\lambda_{l}\right)\left(\prod_{k=1}^{n} x_{k}^{\left\langle m_{1}^{\sigma}, e_{k}\right\rangle}-\lambda_{l+1}\right) \prod_{\tilde{\pi}\left(\rho_{k}\right) \not \subset \sigma} x_{k}} \in R_{1}^{\sigma}(f)_{\beta-\beta_{0}+\beta_{1}^{\sigma}} .
$$

Proof. It suffices to show that the class of $-X_{k, l}+X_{k, l+1}$ in $H^{0,0}\left(X \cap D_{k}\right)$ is represented by the cocycle

$$
\left\{\frac{\left(\lambda_{l+1}-\lambda_{l}\right) f \prod_{k=1}^{n} x_{k}^{\left\langle m_{1}^{\sigma}, e_{k}\right\rangle}\left\langle m_{1}^{\sigma}, e_{i_{0}}\right\rangle}{\left(\prod_{k=1}^{n} x_{k}^{\left\langle m_{1}^{\sigma}, e_{k}\right\rangle}-\lambda_{l}\right)\left(\prod_{k=1}^{n} x_{k}^{\left\langle m_{1}^{\sigma}, e_{k}\right\rangle}-\lambda_{l+1}\right) x_{i_{0}} f_{i_{0}}}\right\}_{i_{0}},
$$

which also represents $\operatorname{Res}_{\rho_{k}}\left(g_{l}\right)$, by Proposition 2.3. Note that

$$
\begin{array}{r}
\left.x_{i_{0}} f_{i_{0}}\right|_{D_{k}}=a_{m_{0}+\operatorname{vol}\left(\Gamma_{\sigma}\right) m_{1}^{\sigma}} x^{D\left(m_{0}\right)}\left(\left(b_{i_{0}}+\left\langle m_{0}, e_{i_{0}}\right) \prod_{s=1}^{\operatorname{vol}\left(\Gamma_{\sigma}\right)}\left(\prod_{k=1}^{n} x_{k}^{\left\langle m_{1}^{\sigma}, e_{k}\right\rangle}-\lambda_{s}\right)\right.\right. \\
\left.+\left\langle m_{1}^{\sigma}, e_{i_{0}}\right\rangle \prod_{k=1}^{n} x_{k}^{\left\langle m_{1}^{\sigma}, e_{k}\right\rangle} \sum_{j=1}^{\operatorname{vol}\left(\Gamma_{\sigma}\right)} \prod_{s \neq j}\left(\prod_{k=1}^{n} x_{k}^{\left\langle m_{1}^{\sigma}, e_{k}\right\rangle}-\lambda_{s}\right)\right) .
\end{array}
$$

Since $\prod_{k=1}^{n} x_{k}^{\left\langle m_{1}^{\sigma}, e_{k}\right\rangle}$ has the value $\lambda_{s}$ on $X_{k, s}$, the above cocycle is equal to -1 on $X_{k, l}, 1$ on $X_{k, l+1}$ and 0 on all other components.

In Section 0 , we will describe the product structure on the ring generated by $\operatorname{Pic}(X)_{\mathbb{C}}$

\section{The B-Model CORRELATion FUnCtions.}

In [M2], we calculated a subring of the B-model chiral ring $H^{*}\left(X, \wedge^{*} \mathcal{T}_{X}\right)$, which contains the space $H^{1}\left(X, \mathcal{T}_{X}\right)$, for semiample anticanonical nondegenerate hypersurfaces. Here, we notice that the ring structure for minimal hypersurfaces is related to a product of the roots of $A$-type Lie algebra. Let us note that a semiample anticanonical nondegenerate hypersurface $X \subset \mathbf{P}_{\Sigma}$ is big and Calabi-Yau. We will use the products on the chiral ring in the next section to propose a mirror map between the space $H^{1}\left(X, \mathcal{T}_{X}\right)$ and the Picard group of the mirror symmetric Calabi-Yau hypersurface $X^{\circ}$ in the Batyrev construction.

We have the birational contraction $\pi: \mathbf{P}_{\Sigma} \rightarrow \mathbf{P}_{\Sigma_{X}}$. In [M2, Section 4], we ordered the cones $\rho_{i}$ inside $\sigma \in \Sigma_{X}(2)$, according the way they lie in $\sigma: \rho_{l_{0}}, \ldots, \rho_{l_{n(\sigma)+1}}$, so that $\rho_{l_{0}}$ and $\rho_{l_{n(\sigma)+1}}$ are the edges of $\sigma$. The corresponding divisors $D_{l_{k}}$ in $\mathbf{P}_{\Sigma}$ intersect only if they are next to each other in the order. Using this order we can state [M2, Theorem 7.1]. 
Theorem 4.1. $\mathrm{M2}$ Let $X \subset \mathbf{P}_{\Sigma}$ be a semiample anticanonical nondegenerate hypersurface defined by $f \in S_{\beta}$. Then there is a natural inclusion

$$
\gamma_{-} \oplus\left(\oplus \gamma_{-}^{\sigma, k}\right): R_{1}(f)_{* \beta} \oplus\left(\bigoplus_{\sigma \in \Sigma_{X}(2)}\left(R_{1}^{\sigma}(f)_{(*-1) \beta+\beta_{1}^{\sigma}}\right)^{n(\sigma)}\right) \hookrightarrow H^{*}\left(X, \wedge^{*} \mathcal{T}_{X}\right)
$$

where the sum $\oplus \gamma_{-}^{\sigma, k}$ is over $\rho_{l_{k}}, k=1, \ldots, n(\sigma)$. Also, $R_{1}^{\sigma}(f)_{(q-1) \beta+\beta_{1}^{\sigma}}=0$ for $q=0, d-1$.

To simplify our further calculations, in this section we make an additional assumption on $\mathbf{P}_{\Sigma}$ :

$$
\operatorname{mult}\left(\sigma^{\prime}\right)=1 \text { for all } \sigma^{\prime} \in \Sigma(2) \text { such that } \sigma^{\prime} \subset \sigma \in \Sigma_{X}(2) \text {. }
$$

In particular, this holds if $X \subset \mathbf{P}_{\Sigma}$ is a minimal Calabi-Yau hypersurface in the mirror construction of $[\mathrm{B}]$. There is a nice way to state the product structure on the subspace of $H^{*}\left(X, \wedge^{*} \mathcal{T}_{X}\right)$ in the above theorem. For this we need to use the $(n(\sigma)+1)$-dimensional vectors $\alpha_{k}=(0, \ldots, 0,-1,1,0, \ldots, 0)$ with -1 on the $k$-th place, which form a basis of the root system of $A_{n(\sigma)}$-type Lie algebra. Also, define the following product of an arbitrary number of vectors $v_{l}=\left(v_{l, 1}, \ldots, v_{l, n(\sigma)+1}\right) \in$ $\mathbb{R}^{n(\sigma)+1}, l=1, \ldots, L$, by the rule:

$$
v_{1} \cdots v_{L}=\sum_{k=1}^{n(\sigma)+1} v_{1, k} \cdots v_{L, k}
$$

As a corollary of [M2, Theorem 6.3], we have:

Theorem 4.2. Let $X \subset \mathbf{P}_{\Sigma}$ be a semiample anticanonical nondegenerate hypersurface defined by $f \in S_{\beta}$, and assume (15) holds. Then, under the identifications of Theorem 4.1, we have

(i) $\gamma_{A} \cup \gamma_{B}=\gamma_{A B}$,

(ii) $\gamma_{A} \cup \gamma_{B}^{\sigma, k}=\gamma_{A B}^{\sigma, k}$,

(iii) $\gamma_{A}^{\sigma_{1}, k_{1}} \cup \gamma_{B}^{\sigma_{2}, k_{2}}=0$ if $\sigma_{1} \neq \sigma_{2}$,

(iv) for $A, B \in R_{1}^{\sigma}(f)_{(*-1) \beta+\beta_{1}^{\sigma}}$, such that $A B \in R_{1}^{\sigma}(f)_{(d-3) \beta+2 \beta_{1}^{\sigma}}$,

$$
\gamma_{A}^{\sigma, k_{1}} \cup \gamma_{B}^{\sigma, k_{2}}=\alpha_{k_{1}} \cdot \alpha_{k_{2}} \gamma_{\mu^{-1}\left(A B G^{\sigma}(f)\right)} \in H^{d-1}\left(X, \wedge^{d-1} \mathcal{T}_{X}\right),
$$

where $\mu: R_{1}(f)_{(d-1) \beta} \rightarrow R_{0}(f)_{d \beta}$ is an isomorphism defined by multiplication with $\prod_{k=1}^{n} x_{k}$, and where $G^{\sigma}(f):=\frac{x_{l_{0}} f_{l_{0}} x_{l_{n(\sigma)+1}} f_{l_{n(\sigma)+1}} \prod_{\rho_{k} \not \subset \sigma} x_{k}}{\operatorname{mult}(\sigma) \prod_{\rho_{k} \subset \sigma} x_{k}} \in S_{3 \beta-2 \beta_{1}^{\sigma}}$,

(v) for $A, B \in R_{1}^{\sigma}(f)_{(*-1) \beta+\beta_{1}^{\sigma}}$, such that $A B C \in R_{1}^{\sigma}(f)_{(d-4) \beta+3 \beta_{1}^{\sigma}}$,

$$
\gamma_{A}^{\sigma, k_{1}} \cup \gamma_{B}^{\sigma, k_{2}} \cup \gamma_{C}^{\sigma, k_{3}}=\alpha_{k_{1}} \cdot \alpha_{k_{2}} \cdot \alpha_{k_{3}} \gamma_{\mu^{-1}\left(A B C H^{\sigma}(f) G^{\sigma}(f)\right)} \in H^{d-1}\left(X, \wedge^{d-1} \mathcal{T}_{X}\right) \text {, }
$$

where

$$
H^{\sigma}(f):=\sqrt{-1} \sum_{m \in \sigma^{\perp} \cap \Delta \cap M} a_{m} \frac{x^{D(m)}}{\prod_{\rho_{k} \subset \sigma} x_{k}} \in S_{\beta-\beta_{1}^{\sigma}}
$$

with $\sigma \in \Sigma_{X}(2), f=\sum_{m \in \Delta \cap M} a_{m} x^{D(m)}$, and the polytope $\Delta$ corresponding to the anticanonical divisor $\sum_{k=1}^{n} D_{k}$.

We can now see a peculiar product structure on a part of the B-model chiral ring $\oplus_{p, q} H^{p}\left(X, \wedge^{q} \mathcal{T}_{X}\right)$. This ring contains modules $\oplus_{q} R_{1}^{\sigma}(f)_{(q-1) \beta+\beta_{1}^{\sigma}}$, corresponding to $e_{k} \in \operatorname{int} \sigma$, over the subring $\oplus_{q} R_{1}(f)_{q \beta}$ called the polynomial part in [M2]. The 
product structure on the sum of these modules is governed by a product of the roots of $A_{n(\sigma)}$-type Lie algebra. While this appears to be surprising, one likely reason for such a mystery comes from the fact that the contraction $Y=\pi(X)$ has $A_{n(\sigma)}$-type singularities along a codimension 2 subvariety $Y \cap V(\sigma)$. The generators $e_{k} \in \operatorname{int} \sigma$ correspond to the exceptional divisors $D_{k}$ of the blow up $\pi$, whose intersection matrix forms the Dynkin diagram of $A_{n(\sigma)}$, as it was observed in KaMoP for the 3-dimensional Calabi-Yau hypersurfaces.

While it is difficult to compute all of the $(d-1)$-point functions of the Calabi-Yau hypersurface, the following products follow from Theorem 4.2 .

Corollary 4.3. Let $X \subset \mathbf{P}_{\Sigma}$ be a semiample anticanonical nondegenerate hypersurface defined by $f \in S_{\beta}$, and assume (15) holds. Given $A_{1}, \ldots, A_{s} \in R_{1}(f)_{\beta}$ and $B_{1}, \ldots, B_{t} \in R_{1}^{\sigma}(f)_{\beta_{1}^{\sigma}}, s+t=d-1, t \leq 3$, then

$$
\gamma_{A_{1}} \cup \cdots \cup \gamma_{A_{s}} \cup \gamma_{B_{1}}^{\sigma, k_{1}} \cup \cdots \cup \gamma_{B_{t}}^{\sigma, k_{t}}=\alpha_{k_{1}} \cdots \alpha_{k_{t}} \gamma_{\mu^{-1}\left(A_{1} \cdots A_{s} B_{1} \cdots B_{t} H^{\sigma}(f)^{t-2} G^{\sigma}(f)\right)} .
$$

This corollary together with the products on the polynomial part $\oplus_{q} R_{1}(f)_{q \beta}$ of the B-model chiral ring, gives all information about the non-normolized 3-point functions (Yukawa couplings) for the Calabi-Yau 3-fold hypersurfaces.

\section{A GeneralizAtion OF THE MONOMiAL-DiVisor MiRROR MAP.}

The monomial-divisor mirror map was proposed in AGM. This map was conjectured to be the derivative of the mirror map at large radius limit points (maximally unipotent boundary points) between the complex and Kähler moduli spaces of a mirror pair $\left(X, X^{\circ}\right)$ of Calabi-Yau hypersurfaces in toric varieties. Here we extend this map to the whole $\operatorname{Pic}(X)_{\mathbb{C}}$ and $H^{1}\left(X^{\circ}, \mathcal{T}_{X^{\circ}}\right)$. Our map is supported by a compatibility of some limiting products of the chiral rings.

While Theorem 2.11 already has a description of the products on the $\operatorname{Pic}(X)_{\mathbb{C}}$, it is very technical to compute $\operatorname{Res}^{\sigma}$ and $p_{\sigma}$ directly. That is why we found an alternative description of the Picard group of $X$ in Corollary 3.3. Using this description we will prove the following.

Proposition 5.1. Let $X \subset \mathbf{P}_{\Sigma}$ be an i-semiample nondegenerate hypersurface defined by $f \in S_{\beta}$. Given $a_{1}, \ldots, a_{s} \in H_{\text {toric }}^{1,1}(X)$, then

$$
a_{1} \cup \cdots \cup a_{s} \cup\left(D_{i_{1}} \otimes g_{k_{1}}\right) \cup \cdots \cup\left(D_{i_{t}} \otimes g_{k_{t}}\right)=\alpha_{k_{1}} \cdots \alpha_{k_{t}} a_{1} \cdots a_{s} \cdot D_{i_{1}} \cdots D_{i_{t}},
$$

where $s+t=d-1$.

Proof. By Lemma 3.4, $D_{i_{j}} \otimes g_{k_{j}}$ corresponds to $-X_{i_{j}, k_{j}}+X_{i_{j}, k_{j}+1}$, which is the same as $\varphi_{\rho_{i_{j}} !} \pi_{\rho_{i_{j}}}^{*}\left(-p_{k_{j}}+p_{k_{j}+1}\right)$. Let us remind that

$$
Y \cap V(\sigma)=\pi(X) \cap V(\sigma)=\bigcup_{k=1}^{\operatorname{vol}\left(\Gamma_{\sigma}\right)} p_{k} .
$$

The product structure on

$$
H^{0}(Y \cap V(\sigma)) \cong \bigoplus_{k=1}^{\operatorname{vol}\left(\Gamma_{\sigma}\right)} \mathbb{C} p_{l}
$$


is an obvious one: multiplication of the corresponding components of the vectors. It is not difficult to see that this product can be written as

$$
\left(-p_{k_{1}}+p_{k_{1}+1}\right) \cup\left(-p_{k_{2}}+p_{k_{2}+1}\right)=\frac{\alpha_{k_{1}} \cdot \alpha_{k_{2}}}{n(\sigma)+1} \sum_{k=1}^{\operatorname{vol}\left(\Gamma_{\sigma}\right)} p_{k}+\sum_{k=1}^{n(\sigma)} c_{k}\left(-p_{k}+p_{k+1}\right)
$$

where the coefficients $c_{k}$ are determined by the decomposition

$$
\left(\alpha_{k_{1}, 1} \alpha_{k_{2}, 1}, \ldots, \alpha_{k_{1}, n(\sigma)+1} \alpha_{k_{2}, n(\sigma)+1}\right)=\frac{\alpha_{k_{1}} \cdot \alpha_{k_{2}}}{n(\sigma)+1}(1, \ldots, 1)+\sum_{k=1}^{n(\sigma)} c_{k} \alpha_{k}
$$

Hence,

$$
\begin{array}{r}
\left(D_{i_{1}} \otimes g_{k_{1}}\right) \cup\left(D_{i_{2}} \otimes g_{k_{2}}\right)=\varphi_{\rho_{i_{1} !}} \pi_{\rho_{i_{1}}}^{*}\left(-p_{k_{1}}+p_{k_{1}+1}\right) \cup \varphi_{\rho_{i_{2}} !} \pi_{\rho_{i_{2}}}^{*}\left(-p_{k_{2}}+p_{k_{2}+1}\right) \\
=i^{*} D_{i_{2}} \cup \varphi_{\rho_{i_{1}} !} \pi_{\rho_{i_{1}}}^{*}\left(\left(-p_{k_{1}}+p_{k_{1}+1}\right) \cup\left(-p_{k_{2}}+p_{k_{2}+1}\right)\right) \\
=\frac{\alpha_{k_{1}} \cdot \alpha_{k_{2}}}{n(\sigma)+1} D_{i_{1}} D_{i_{2}}+\sum_{k=1}^{n(\sigma)} c_{k}\left(D_{i_{1}} D_{i_{2}} \otimes g_{k}\right)
\end{array}
$$

where we also use the same technique as in the proof of Proposition 2.9.

Now we can prove the proposition by induction on $t$. It is true for $t=1$ : the left side of the formula vanishes because the residue part is orthogonal to the toric part, while the other side also vanishes by our definition of the products of the vectors. Assuming that the statement holds for $t-1$, we have

$$
\begin{aligned}
& a_{1} \cup \cdots \cup a_{s} \cup\left(D_{i_{1}} \otimes g_{k_{1}}\right) \cup \cdots \cup\left(D_{i_{t}} \otimes g_{k_{t}}\right) \\
= & a_{1} \cup \cdots \cup a_{s} \cup\left(\frac{\alpha_{k_{1}} \cdot \alpha_{k_{2}}}{n(\sigma)+1} D_{i_{1}} D_{i_{2}}+\sum_{k=1}^{n(\sigma)} c_{k}\left(D_{i_{1}} D_{i_{2}} \otimes g_{k}\right)\right) \cup\left(D_{i_{3}} \otimes g_{k_{3}}\right) \cup \cdots \cup\left(D_{i_{t}} \otimes g_{k_{t}}\right) \\
= & \frac{\alpha_{k_{1}} \cdot \alpha_{k_{2}}}{n(\sigma)+1} a_{1} \cdots a_{s} D_{i_{1}} D_{i_{2}} \cdots D_{i_{t}}+\sum_{k=1}^{n(\sigma)} c_{k} \alpha_{k} \cdot \alpha_{k_{3}} \cdots \alpha_{k_{t}} a_{1} \cdots a_{s} D_{i_{1}} D_{i_{2}} \cdots D_{i_{t}} \\
= & \alpha_{k_{1}} \cdots \alpha_{k_{t}} a_{1} \cdots a_{s} \cdot D_{i_{1}} \cdots D_{i_{t}} .
\end{aligned}
$$

We can see a similarity between the products in the above proposition and Corollary 4.3. Based on this we will propose a mirror map between $\operatorname{Pic}(X)_{\mathbb{C}}$ and $H^{1}\left(X^{\circ}, \mathcal{T}_{X^{\circ}}\right)$ for a mirror pair $\left(X, X^{\circ}\right)$ of Calabi-Yau hypersurfaces.

Let us recall the mirror symmetry construction from $[\mathrm{B}]$. We will describe it starting with a semiample nondegenerate Calabi-Yau hypersurface $X$ in a complete simplicial toric variety $\mathbf{P}_{\Sigma}$. Such a hypersurface has the anticanonical degree $\left[\sum_{i=1}^{n} D_{n}\right]$. Theorem 1.2 gives a unique birational toric morphism $\pi: \mathbf{P}_{\Sigma} \rightarrow \mathbf{P}_{\Sigma_{X}}$ such that the fan $\Sigma$ is a subdivision of the fan $\Sigma_{X}, \pi_{*}[X]$ is ample and $\pi^{*} \pi_{*}[X]=$ $[X]$. Moreover, $\Sigma_{X}$ is the normal fan of the polytope $\Delta$ associated with a torus invariant divisor equivalent to $X$. The push-forward $\pi_{*}[X]$ is again anticanonical, whence the toric variety $\mathbf{P}_{\Sigma_{X}}$ is Fano associated with the polytope $\Delta \subset M_{\mathbb{R}}$ of the anticanonical divisor $\sum_{i=1}^{n} D_{n}$ on $\mathbf{P}_{\Sigma}$, by Lemma 3.5.2 in CKa. Then, Proposition 1.6 shows that the image $Y:=\pi(X)$ is an ample nondegenerate hypersurface in $\mathbf{P}_{\Sigma_{X}}=\mathbf{P}_{\Delta}$. The fact that $\mathbf{P}_{\Delta}$ is Fano means by Proposition 3.5.5 in [CKa that the polytope $\Delta$ is reflexive, i.e., its dual

$$
\Delta^{\circ}=\left\{n \in N_{\mathbb{R}}:\langle m, n\rangle \geq-1 \text { for } m \in \Delta\right\}
$$


has all its vertices at lattice points in $N$, and the only lattice point in the interior of $\Delta^{\circ}$ is the origin 0 . This implies that the generators of the 1-dimensional cones of the fan $\Sigma$ are among the lattice points of $\Delta^{\circ}$. If $X$ was a minimal Calabi-Yau (see CKa ), then these generators coincide with the set $\Delta^{\circ} \cap N \backslash\{0\}$. Now, consider the polytope $\Delta^{\circ}$ and the associated toric variety $\mathbf{P}_{\Delta^{\circ}}$. Theorem 4.1.9 in $\mathrm{B}$ says that an anticanonical nondegenerate hypersurface $Y^{\circ} \subset \mathbf{P}_{\Delta^{\circ}}$ is a Calabi-Yau variety with canonical singularities. To obtain a mirror of $X$, it is natural to take a certain desingularization of $Y^{\circ}$. Batyrev introduced the following notion.

Definition 5.2. A projective birational morphism $\varphi: W^{\prime} \rightarrow W$ is called a maximal projective crepant partial desingularization (MPCP-desingularization) of $W$ if $\varphi$ is crepant $\left(\varphi^{*}\left(K_{W}\right)=K_{W^{\prime}}\right)$ and $W^{\prime}$ has only $\mathbb{Q}$-factorial terminal singularities.

Take any simplicial subdivision $\Sigma^{\circ}$ of the normal fan of $\Delta^{\circ}$ such that the generators of the 1-dimensional cones of $\Sigma^{\circ}$ is the set $\Delta \cap M \backslash\{0\}$. Such a subdivision corresponds to a toric birational morphism $\mathbf{P}_{\Sigma^{\circ}} \rightarrow \mathbf{P}_{\Delta^{\circ}}$ which is a MPCPdesingularization of $\mathbf{P}_{\Delta^{\circ}}$, by Lemma 4.1.2 in CKa. Theorem 2.2.24 of B] says that the toric variety $\mathbf{P}_{\Delta}$ 。 admits at least one MPCP-desingularization. This also induces a MPCP-desingularization $X^{\circ} \subset \mathbf{P}_{\Sigma^{\circ}}$ of the anticanonical hypersurface $Y^{\circ} \subset \mathbf{P}_{\Delta}$. The hypersurface $X^{\circ}$ is a minimal Calabi-Yau variety. Batyrev showed the following formulas for some Hodge numbers of the minimal Calabi-Yau hypersurfaces (MPCP-desingularizations):

$$
h^{d-2,1}\left(X^{\circ}\right)=h^{1,1}(X)=l\left(\Delta^{\circ}\right)-d-1-\sum_{\operatorname{codim} \theta=1} l^{*}(\theta)+\sum_{\operatorname{codim} \theta=2} l^{*}(\theta) l^{*}\left(\theta^{*}\right),
$$

where $\theta$ is a face of $\Delta^{\circ}, \theta^{*}$ is the corresponding dual face of the dual reflexive polyhedron $\Delta$, and $l(\Gamma)$ (resp., $l^{*}(\Gamma)$ ) denotes the number of lattice (resp., interior lattice) points in $\Gamma$. For Calabi-Yau 3-folds, this is called the topological mirror symmetry test. Note also that since $X^{\circ}$ is Calabi-Yau, $h^{d-2,1}\left(X^{\circ}\right)=\operatorname{dim} H^{1}\left(X^{\circ}, \mathcal{T}_{X^{\circ}}\right)$.

The monomial-divisor mirror map can be easily described if we assume that the polynomial $f \in S_{\beta}$ defining the hypersurface $X^{\circ} \subset \mathbf{P}_{\Sigma^{\circ}}$ has the form

$$
\sum_{m \in\left(\Delta^{\circ} \cap M\right)_{0}} a_{m} x^{D(m)}
$$

where $\left(\Delta^{\circ} \cap M\right)_{0}$ is the set of all lattice points in $\Delta^{\circ}$ which do not lie in the interior of any facet of $\Delta^{\circ}$. Such a hypersurface has been called simplified in AGM, CKa. In this case, the map between the toric part $H_{\text {toric }}^{1,1}(X)$ and the polynomial part $R_{1}(f)_{\beta}$ of $H^{1}\left(X^{\circ}, \mathcal{T}_{X^{\circ}}\right)$ is defined by sending the divisor $D_{k}$, corresponding to the lattice point $e_{k}$ in $\left(\Delta^{\circ} \cap M\right)_{0}$, to the monomial $x^{D\left(e_{k}\right)}$. One can verify that this map is well defined and induces an isomorphism. We should note that if the hypersurface is not simplified the mirror map is complicated to describe explicitly.

Our extension of the monomial-divisor mirror map is as follows. Assign to the divisors $-X_{k, l}+X_{k, l+1}=D_{k} \otimes g_{l} \in H^{1,1}(X)$, for $e_{k} \in \operatorname{int} \sigma, \sigma \in \Sigma_{X}(d-1)$ and $l=1, \ldots, \operatorname{vol}\left(\Gamma_{\sigma}\right)-1$, the elements $\gamma_{A}^{\sigma^{\circ}, l}$ with

$$
A=\frac{x^{D\left(e_{k}\right)}}{\prod_{\rho_{k} \not \subset \sigma} x_{k}} \in R_{1}^{\sigma^{\circ}}(f)_{\beta_{1}^{\sigma^{\circ}}}
$$

where $\sigma^{\circ} \in \Sigma_{X^{\circ}}(2)$ is the cone over the 1-dimensional face $\Gamma_{\sigma}$. Using the formula for the Hodge numbers and comparing the dimensions, one deduces that $\operatorname{dim} R_{1}^{\sigma^{\circ}}(f)_{\beta_{1}^{\sigma^{\circ}}}$ coincides with the number of $e_{k} \in \operatorname{int} \sigma$. Therefore, our map is an 
isomorphism. We conjecture that this map is the derivative of the mirror map at the large radius limit points of the Kähler and complex moduli spaces of the pair of Calabi-Yau hypersurfaces. One consequence of mirror symmetry is that the derivative of the mirror map between the moduli spaces gives a ring isomorphism between the quantum cohomology of $X$ and the B-model chiral ring of $X^{\circ}$ for a mirror pair of smooth Calabi-Yau hypersurfaces. In particular, the compatibility of the products should also be valid at the limit points. The limit of the product of the quantum cohomology ring is the usual cup product on the cohomology. On the other hand, we have the products of the B-model chiral ring in Corollary 1.3 . While it is very technical to compute explicitly the limits of non-vanishing products, the limits of the vanishing products are zero. The products in Corollary 4.3 vanish when there are 2 non-consecutive numbers among $k_{1}, \ldots, k_{t}$ or if $k_{1}, \ldots, k_{t}$ are the same number and $t$ is odd. The same holds for the products of the cohomology ring in Proposition 5.1. This verifies the compatibility of our generalization of the monomial-divisor mirror map with some of the limiting products.

Since the vanishing products hold on the B-model chiral ring far from the limiting points in the complex moduli space, we believe that a similar statement should be true for the quantum products of the mirror Calabi-Yau hypersurface. Thus, we conjecture that

$$
\begin{aligned}
& a_{1} * \cdots * a_{s} *\left(D_{i_{1}} \otimes g_{k_{1}}\right) * \cdots *\left(D_{i_{t}} \otimes g_{k_{t}}\right) \\
& \quad=a_{1} * \cdots * a_{s} *\left(-X_{i_{1}, k_{1}}+X_{i_{1}, k_{1}+1}\right) * \cdots *\left(-X_{i_{t}, k_{t}}+X_{i_{t}, k_{t}+1}\right)=0
\end{aligned}
$$

where $*$ denotes the small quantum product on $H^{*}(X)$ (see CKa $)$, if $\left|k_{a}-k_{b}\right|>1$ for some $a, b$ or if $k_{1}=\cdots=k_{t}$ and $t$ is odd.

\section{REFERENCES}

[AGM] P. Aspinwall, B. Greene, D. Morrison, The monomial-divisor mirror map, Internat. Math. Res. Notices, no. 12 (1993), 319-337.

[B] V. V. Batyrev, Dual polyhedra and mirror symmetry for Calabi-Yau hypersurfaces in toric varieties, J. Algebraic Geometry 6 (1994), 493-535.

[BC] V. V. Batyrev and D. A. Cox, On the Hodge structure of projective hypersurfaces in toric varieties, Duke Math. J. 75 (1994), 293-338.

[CaG] J. Carlson and P. Griffiths, "Infinitesimal variations of Hodge structure and the global Torelli problem" in Journées de Géométrie Algébrique d'Angers, juillet 1979, Sijthoff and Nordhoff, Alphen aan den Rijn, Germantown, Md., 1980, pp. 51-76.

[C] D. A. Cox, Toric residues, Ark. Mat., 34 (1996), 73-96.

[CKa] D. Cox and S. Katz, Algebraic Geometry and Mirror Symmetry, Math. Surveys Monogr. 68, Amer. Math. Soc., Providence, 1999.

[D] V. Danilov, The geometry of toric varieties, Russian Math. Surveys 33 (1978), 97-154.

[DK] V. Danilov and A. Khovanskii, Newton polyhedra and an algorithm for computing Hodge-Deligne numbers, Math. USSR-Izv. 29 (1987), 279-298.

[F] W. Fulton, Introduction to toric varieties, Princeton Univ. Press, Princeton, NJ, 1993.

[G] P. Griffiths, On the periods of certain rational integrals I, II, Ann. of Math. (2) 90 (1969) 460-495, 498-541.

[KaMoP] S. Katz, D. Morrison, and M. R. Plesser, Enhanced Gauge Symmetry in Type II String Theory, Nuclear Phys. B 477 (1996), no. 1, 105-140.

[M1] A. R. Mavlyutov, Semiample hypersurfaces in toric varieties, Duke Math. J. 101 (2000), 85-116.

[M2] , "On the chiral ring of Calabi-Yau hypersurfaces in toric varieties", preprint /math.AG/0010318

[M3] - Toric Geometry and Mirror Symmetry, Ph.D. Dissertation, University of Massachusetts at Amherst, 2000. 
[R] S-S. Roan, Picard groups of hypersurfaces in toric varieties, Publ. Res. Inst. Math. Sci. 32 (1996), 797-834.

[V] C. Vafa, String vacua and orbifoldized LG models, Modern Phys. Lett., A4 (1989), no. $12,1169-1185$.

Max-Planck-Institut für Mathematik, Bonn, D-53111, Germany.

E-mail address: anvar@mpim-bonn.mpg.de 